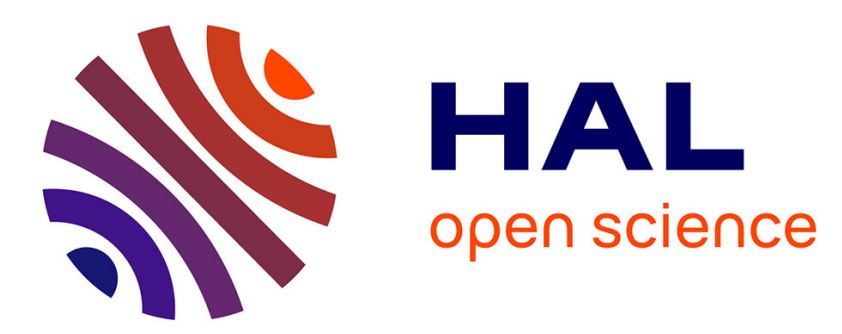

\title{
The onset of three-dimensional centrifugal global modes and their nonlinear development in a recirculating flow over a flat surface
}

Stefania Cherubini, Jean-Christophe Robinet, Pietro de Palma, Frédéric

Alizard

\section{To cite this version:}

Stefania Cherubini, Jean-Christophe Robinet, Pietro de Palma, Frédéric Alizard. The onset of threedimensional centrifugal global modes and their nonlinear development in a recirculating flow over a flat surface. Physics of Fluids, 2010, 22 (114102), pp.18. 10.1063/1.3500677 . hal-00799785

\section{HAL Id: hal-00799785 \\ https://hal.science/hal-00799785}

Submitted on 12 Mar 2013

HAL is a multi-disciplinary open access archive for the deposit and dissemination of scientific research documents, whether they are published or not. The documents may come from teaching and research institutions in France or abroad, or from public or private research centers.
L'archive ouverte pluridisciplinaire HAL, est destinée au dépôt et à la diffusion de documents scientifiques de niveau recherche, publiés ou non, émanant des établissements d'enseignement et de recherche français ou étrangers, des laboratoires publics ou privés. 


\title{
The onset of three-dimensional centrifugal global modes and their nonlinear development in a recirculating flow over a flat surface
}

\author{
S. Cherubini, ${ }^{1,2}$ J.-Ch. Robinet, ${ }^{2}$ P. De Palma, ${ }^{1}$ and F. Alizard ${ }^{2}$ \\ ${ }^{1}$ DIMeG, CEMeC, Politecnico di Bari, Via Re David 200, 70125 Bari, Italy \\ ${ }^{2}$ DynFluid Laboratory, Arts et Metiers ParisTech, 151 Bd. de l'Hôpital, 75013 Paris, France
}

(Received 17 February 2010; accepted 3 August 2010; published online 2 November 2010)

\begin{abstract}
The three-dimensional stability dynamics of a separation bubble over a flat plate has been studied in both linear and nonlinear conditions. Using a global eigenvalue analysis, two centrifugal global modes are identified: an asymptotically unstable three-dimensional weakly growing mode which appears to be originated by a Rayleigh instability; a marginally stable three-dimensional steady mode which is originated by a convective Gortler instability. Direct numerical simulations show that both modes play a role in the route to transition toward the turbulent flow. A structural sensitivity analysis is used to investigate the mechanism of selection of the path toward transition when small perturbations are considered. Finally, a scenario of transition via Gortler modes breakdown is studied in detail, revealing the formation of trains of hairpin vortices in streamwise succession.

(C) 2010 American Institute of Physics. [doi:10.1063/1.3500677]
\end{abstract}

\section{INTRODUCTION}

This paper aims at investigating the base mechanisms of the transition to turbulence for a fundamental flow such as the recirculating boundary layer over a flat plate. Recently, new ideas have been developed to understand the instability patterns affecting simple base flows at transitional Reynolds numbers $(\mathrm{Re})$. The motivation is that, in some cases, the classical linear modal analysis fails to predict the critical values of $\mathrm{Re}$ which are experimentally found to be smaller than the ones provided by the theory. ${ }^{1}$ The main reason of this discrepancy is that early theoretical achievements were based on the simplifying assumption that the instability dynamics is driven by the asymptotic exponential growth of small perturbations. Exponentially growing modes were identified by an eigenvalue analysis based on the hypothesis of parallel flow (with one eigendirection normal to the wall), which is a satisfactory assumption only when the streamwise length scale of the variations of the base flow is small with respect to the wavelength of the perturbation. ${ }^{2}$ The limitations of such an approach, known as local modal analysis, were recognized in the late 1980 s, when the concept of nonnormality of the linearized Navier-Stokes operator ${ }^{3,4}$ was introduced and linked to the physical behavior of the flow through the lift-up mechanism..$^{5}$ This new approach concentrated on the analysis of the evolution of disturbances at short times, when the receptivity of an (asymptotically) stable flow, namely, its capability of amplifying the perturbation in a finite interval of time, may induce weakly nonlinear interactions which lead to transition. ${ }^{1}$

When the flow is far from being parallel, a global approach is needed, the perturbation behavior being determined by the "competition" between different zones of convective and absolute instability (see Ref. 2 and references therein). When an open flow is linearly globally stable while being convectively unstable, it behaves as an amplifier of external perturbations. In this case, disturbances are continuously am- plified while being convected away from the unstable region. On the other hand, when the flow is linearly globally unstable, an absolute instability region induces self-sustained perturbations and the flow acts as a resonator. The global approach was firstly employed to study the vortex shedding in flows past bluff bodies ${ }^{6,7}$ and usually consists in assuming two eigendirections (normal to the wall and streamwise) with exponential time evolution, so that the resulting eigenvalue problem provides more accurate two-dimensional information about the (asymptotically) unstable modes. However, the information contained in the global spectrum of a differential operator for a given base flow is not sufficient to characterize the flow dynamics when the operator is non-normal. In fact, in this case, the global eigenmodes are nonorthogonal and their interactions may lead to strong transient amplifications. The global approach may also be employed for studying the transient growth, since the perturbation inducing the largest growth in the energy gain may be approximated by the superposition of global eigenmodes. This approach has been recently employed to study the transient growth for several base flows including recirculating flows generated by the geometry $^{8,9}$ or by an adverse pressure gradient over a flat plate. ${ }^{10}$ In Refs. 11 and 12, a direct-adjoint method is employed to perform the global transient growth analysis, thus avoiding the approximation based on the use of a finite number of modes. By means of this method, the stability of geometry-induced recirculating flows has been studied, investigating both amplifier and resonator dynamics. Indeed, both dynamics play an important role in the instability evolution of recirculating flows, the former being associated with nearly two-dimensional unsteady structures, whereas the latter with three-dimensional steady ones.

Nevertheless, it is still not clear which is the origin of such a resonator instability and under which conditions it arises in the case of separated flows. In Ref. 13, the authors experimentally observed a three-dimensional resonator dynamics on a flow past a backward-facing step, linking it to 
the onset of a Gortler-type mechanism. Evidence of a Gortler modal instability has been recently found by an adjointbased spatial theory and confirmed by experiments and direct numerical simulations (DNSs). ${ }^{14}$ On the other hand, it has been proved the existence of an intrinsic three-dimensional centrifugal instability mechanism originating on the closed streamlines within the separation bubble past a bump ${ }^{15}$ and a backward-facing step. ${ }^{16}$ Moreover, the analysis of the direct and adjoint global modes in an s-shaped channel, carried out in Ref. 17, has linked the resonator dynamics to the lift-up mechanism. Indeed, the overlapping region between the adjoint and the direct global modes has been recognized to have a large impact on the eigenvalues of the global spectrum. ${ }^{2}$ In Ref. 18, this region has been identified as the wave-maker one, namely, the region where the core of the instability is located. This structural sensitivity analysis provides a new way to investigate the properties and the structure of global modes. Even if several explanations have been proposed, it is still not clear which is the real origin of the three-dimensionality arising asymptotically in separated flows, and which is the role of the resonator dynamics in the route to transition. In particular, the interaction and competition of amplifier and resonator instabilities in separated flows have been not investigated so far in a nonlinear framework, and it is still an unresolved issue whether and under which conditions the strong amplifier two-dimensional instability is able to bypass the asymptotic mechanism.

The aim of this paper is to study the mechanisms leading to the amplifier and the resonator dynamics in a recirculation bubble induced by an adverse pressure gradient over a flat plate. The focus will be on the onset of different threedimensional centrifugal modes and their role in transition. This work follows the two-dimensional global analysis of Ref. 10, in which the instability mechanisms induced by nonnormality, such as the flapping phenomenon, were investigated. The three-dimensional global stability analysis is applied by considering two eigendirections and a wave-like solution in the spanwise direction. Such a technique is described in Sec. III, together with the numerical method employed for the DNS. In Sec. IV A, the most relevant global modes of the spectrum are identified. Then, the origin of the two fundamental steady modes is discussed, demonstrating their centrifugal nature; the Rayleigh criterion and the Gortler analysis are employed at this purpose. The transient amplification of the perturbation is also investigated with respect to the variation of the spanwise wavenumber. In Secs. IV B and IV C, DNS is used to study the nonlinear effects which influence the evolution of small-amplitude and large-amplitude perturbations. The relevant global modes are identified and extracted from the DNS at different times. In order to understand the mechanism of selection of the path toward transition, a structural sensitivity analysis of the base flow is employed. Therefore, it is possible to identify the regions of the flow generating the resonator and the amplifier behavior and to describe a possible scenario of the transition process which involves the interaction of the two main centrifugal global modes.

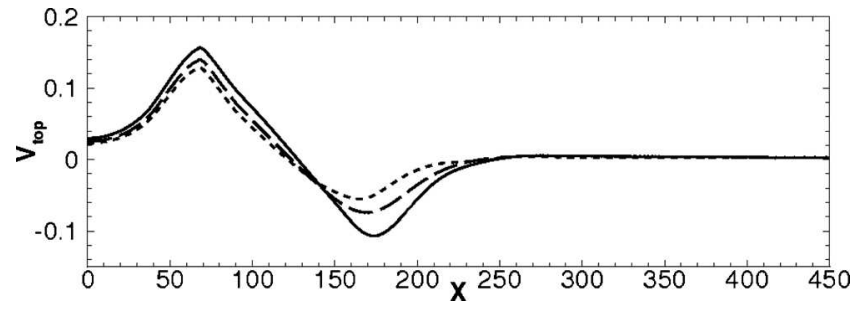

FIG. 1. Suction-and-blowing profiles imposed at the upper boundary for the wall-normal component of the velocity corresponding to base flows BF1 (solid line), BF2 (long-dashed line), and BF3 (short-dashed line).

\section{GOVERNING EQUATION AND BOUNDARY CONDITIONS}

The three-dimensional incompressible flow over a flat plate has been computed by solving the Navier-Stokes equations,

$$
\begin{aligned}
& \mathbf{u}_{t}+(\mathbf{u} \cdot \nabla) \mathbf{u}=-\nabla \pi+\frac{1}{\operatorname{Re}} \nabla^{2} \mathbf{u}, \\
& \nabla \cdot \mathbf{u}=0,
\end{aligned}
$$

where $\mathbf{u}$ is the velocity vector and $\pi$ is the pressure. Dimensionless variables are defined with respect to the inflow displacement thickness $\delta^{k}$ and to the freestream velocity $U_{\infty}$, so that the Reynolds number is equal to $\operatorname{Re}=U_{\infty} \delta^{*} / \nu$, where $\nu$ is the kinematic viscosity coefficient. A rectangular computational domain is employed, having dimensions $L_{x}=425$, $L_{y}=30, L_{z}=62.8, x, y$, and $z$ being the streamwise, wallnormal, and spanwise directions, respectively. At inlet points, placed at $x_{\mathrm{in}}=65$ from the leading edge of the bottom wall, the Blasius boundary-layer profile is imposed for the streamwise and wall-normal components of the velocity vector, and the spanwise component is set to zero. At outlet points, a standard convective condition is employed. At the bottom wall, the no-slip boundary condition is prescribed. A suctionand-blowing profile for the wall-normal component of the velocity ${ }^{19}$ is imposed at the upper-boundary points: three profiles with different magnitude, associated with base flows $\mathrm{BF} 1, \mathrm{BF} 2$, and BF3, have been considered, see Fig. 1. Furthermore, the spanwise velocity component and the vorticity are set to zero. Finally, in the spanwise direction, periodicity is imposed for the three velocity components.

\section{NUMERICAL METHODS}

\section{A. DNS}

The Navier-Stokes equations are integrated by a fractional step method using a staggered grid. ${ }^{20}$ The viscous terms are discretized in time using an implicit CrankNicholson scheme, whereas an explicit third-order-accurate Runge-Kutta scheme is employed for the nonlinear terms. A second-order-accurate centered space discretization has been used for the spatial discretization.

All numerical simulations provided in the present work have been performed discretizing the computational domain by a Cartesian grid stretched in the wall-normal direction. The computational domain contains $501 \times 150 \times 81$ grid 
points, the height of the first cell close to the wall being equal to 0.1. A numerical grid-convergence study for the separated flow under consideration is provided in Ref. 10.

\section{B. Global eigenvalue analysis}

Once the base flows have been computed by the DNS, their global stability is studied by means of a perturbative technique, namely, by considering the instantaneous variables as a superposition of the base flow $\mathbf{U}=(U, V, 0, P)^{T}$ and of the perturbation $\mathbf{q}=(u, v, w, p)^{T}$. The perturbation is supposed to be characterized by only one wavenumber $\beta$ in the spanwise direction, and it is decomposed in temporal modes as

$$
\mathbf{q}(x, y, z, t)=\sum_{k=1}^{N} \kappa_{k}^{0} \hat{\mathbf{q}}_{k}(x, y) \exp \left(-i \omega_{k} t+i \beta z\right),
$$

where $N$ is the total number of modes, $\hat{\mathbf{q}}_{k}$ are the eigenvectors, $\omega_{k}$ are the eigenmodes (complex frequency), and $\kappa_{k}^{0}$ represents the initial energy of each mode. Substituting such a decomposition in Eq. (1) and linearizing, the following eigenvalue problem is obtained:

$$
\left(\mathbf{A}-i \omega_{k} \mathbf{B}\right) \hat{\mathbf{q}}_{k}=0, \quad k=1, \ldots, N,
$$

where matrices $\mathbf{A}$ and $\mathbf{B}$ are defined in Appendix A.

In order to quantify the amplification of the perturbation, the disturbance energy density is defined as

$$
E(t)=\int_{0}^{L_{z}} \int_{0}^{L_{y}} \int_{x_{\mathrm{in}}}^{x_{\mathrm{out}}}\left(u^{2}+v^{2}+w^{2}\right) d x d y d z .
$$

Furthermore, the maximum energy gain obtainable at time $t$ over all possible initial conditions $\mathbf{q}_{0}$ is

$$
G(t)=\max _{\mathbf{q}_{0} \neq 0} \frac{E(t)}{E(0)} .
$$

By decomposing the perturbation into the eigenmode basis (2), it is possible to rewrite Eq. (5) in the following form:

$$
G(t)=\left\|\mathbf{F} \exp (-i t \mathbf{\Lambda}) \mathbf{F}^{-1}\right\|_{2}^{2},
$$

where $\boldsymbol{\Lambda}$ is the diagonal matrix of the eigenvalues $\omega_{k}$, and $\mathbf{F}$ is the Cholesky factor of the energy matrix $\mathbf{M}$ of components

$$
\begin{aligned}
& M_{i j}=\int_{0}^{L_{y}} \int_{x_{\mathrm{in}}}^{x_{\mathrm{out}}}\left(\hat{u}_{i}^{*} \hat{u}_{j}+\hat{v}_{i}^{*} \hat{v}_{j}+\hat{w}_{i}^{*} \hat{w}_{j}\right) d x d y, \\
& i, j=1, \ldots, N,
\end{aligned}
$$

where the superscript $*$ denotes the complex-conjugate. Finally, the maximum amplification at time $t$ and the corresponding optimal initial condition $\mathbf{q}_{0}$ are computed by a singular value decomposition of the matrix $\mathbf{F} \exp (-i t \mathbf{\Lambda}) \mathbf{F}^{-1}$. $^{21}$

Problem (3) is discretized with a Chebyshev/Chebyshev collocation spectral method employing $N=1500$ modes, and is solved with a shift-and-invert Arnoldi algorithm using the ARPACK library, ${ }^{22}$ the residual being reduced to $10^{-12}$. At upper and inlet boundary points, a zero perturbation condition is imposed, whereas at the outflow, a Neumann condition is prescribed. The base flow is discretized using $N_{x}$

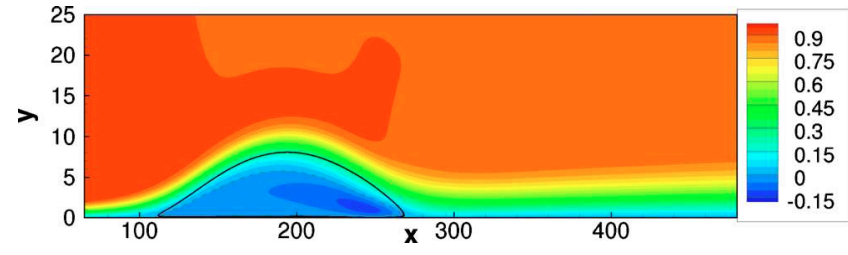

FIG. 2. (Color online) Streamwise velocity contours for the base flow BF1 at $\operatorname{Re}=200$. The solid line is the separation streamline, whereas the dashed line represents the $u=0$ contour.

$=230$ collocation points in the $x$-direction and $N_{y}=47$ collocation points in the $y$-direction. A numerical convergence study is provided in Ref. 10.

\section{FLOW ANALYSIS}

\section{A. Linear dynamics using the global eigenvalue analysis}

\section{Spectrum analysis}

Unless otherwise stated, the computations in this section have been performed for BF1. Figure 2 shows the corresponding streamwise velocity contours at $\operatorname{Re}=200$. For such a base flow and $\beta=0.1$, the global eigenvalue analysis provides the spectrum shown in Figure 3, which is found to be unstable. Different families of modes, identified on the basis of the physical character and shape of the eigenmodes, can be detected, some of them having a very low growth rate. The three most amplified families, enclosed in the dashed rectangles in Fig. 3, are considered here. Two modes for each family are analyzed, namely, $M_{S}$ and $M_{G}$ for the steadymode family; $M_{\mathrm{US}_{1}}$ and $M_{\mathrm{US}_{2}}$, placed on the branch of the unsteady low-frequency modes; $M_{\mathrm{TS}_{1}}$ and $M_{\mathrm{TS}_{2}}$, placed on the branch of the high-frequency ones. All of these modes are found to be relevant in the dynamics of the flow, as shown in section IV B.

The asymptotic behavior of the flow is driven by the unstable mode labeled $M_{S}$ in Fig. 3, which is a threedimensional steady mode $\left(\omega_{r}=0\right)$. Such a mode is similar to the unstable weakly growing one found in Ref. 23 for a three-dimensional separated flow induced by an adverse pressure gradient and to the ones recovered in Refs. 16 and 17 for the case of geometry-induced separated flows. The contours of the streamwise and spanwise velocity components of such a mode can be observed in Figs. 4(a) and 4(b), respectively, showing that a great part of the perturbation is concentrated within the bubble. Figure 4(c) provides the contours of the local energy, defined as $e=u^{2}+v^{2}+w^{2}$; one can notice that the energy is concentrated within the recirculation

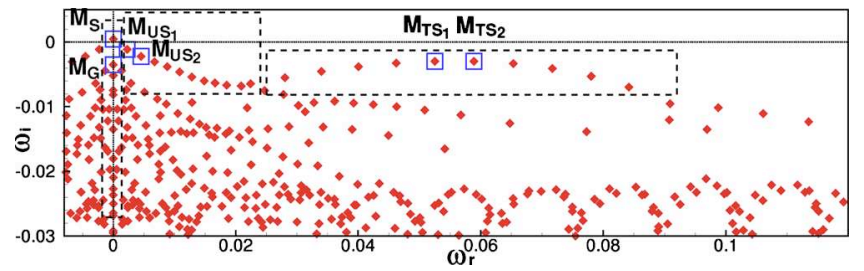

FIG. 3. (Color online) Spectrum associated with the base flow BF1 for $\beta=0.1$. The dashed rectangles identify three families of modes. 

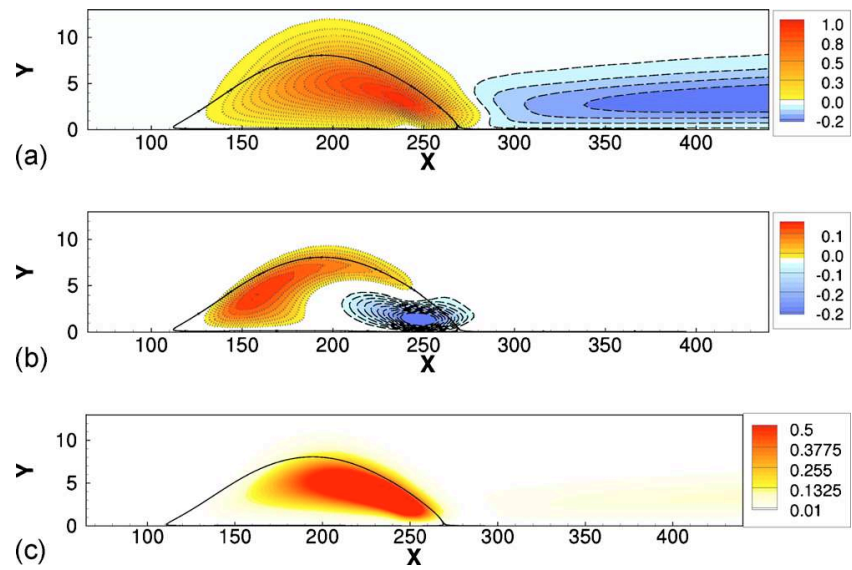

FIG. 4. (Color online) Contours of the (a) streamwise and (b) spanwise perturbations (normalized by the maximum value of the streamwise one) and of the (c) local energy $e$, (normalized by its maximum value) for the unstable steady mode $\left(M_{S}\right)$ at $\operatorname{Re}=200$. The black line is the separation streamline, whereas dashed contours represent positive perturbations and dotted contours represent negative ones.

zone. In the spanwise direction, the mode $M_{S}$ takes the form of alternated flat roll structures which follow the curvature of the streamlines of the base flow, as shown by the spanwise vorticity isosurfaces in Fig. 5(a). Similar modes have been recovered in several separated flows induced by the geometry of the flow, such as a step ${ }^{16,17,24}$ or a bump. ${ }^{15}$ The marginally stable steady mode is the one labeled $M_{G}$ in Fig. 3. Figure 5(b) shows that its spanwise vorticity distribution presents some similarities with the one of mode $M_{S}$, being characterized by flat roll structures following the curvature of the
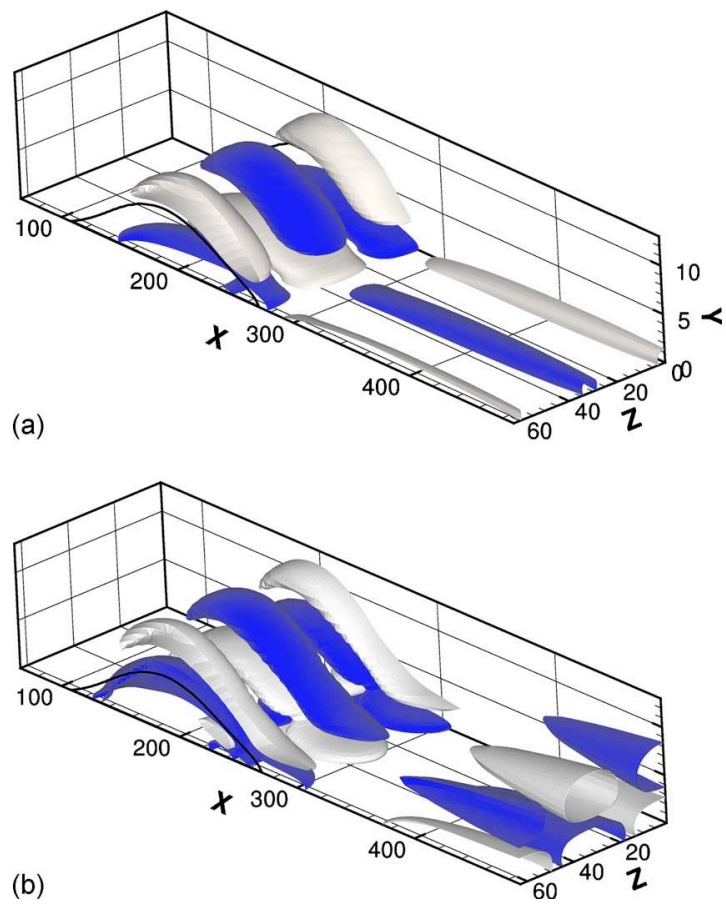

FIG. 5. (Color online) Isosurfaces of spanwise vorticity of the perturbation for the most amplified steady modes (a) $M_{S}$ and (b) $M_{G}$ at $\operatorname{Re}=200$. The black line is the separation streamline, whereas the dark and light surfaces represent the 0.15 and -0.15 values, respectively. The perturbations have been normalized by their maximum value.

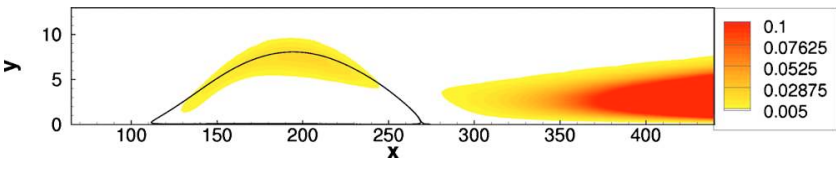

FIG. 6. (Color online) Local energy of the steady mode $M_{G}$ at $\operatorname{Re}=200$. The black line is the separation streamline.

streamlines. Therefore, it can be supposed that both these three-dimensional global modes could have a common origin, which could be linked to a centrifugal instability, ${ }^{15}$ being both strongly affected by the curvature of the streamlines. On the other hand, the two modes present some differences, as shown in Fig. 6, which provides the local energy contours of mode $M_{G}$. By comparing such a figure with Fig. 4(c), one can observe that a great part of the energy is concentrated in the attached zone and in the shear layer; not within the bubble as it has been previously found for mode $M_{S}$. Thus, $M_{S}$ is mainly located inside the bubble, whereas $M_{G}$ has a strong spatial development, as one can also observe by comparing Figs. 5(a) and 5(b).

The second family of modes is characterized by the lowfrequency unsteady eigenvalues shown in the second (from left to right) dashed rectangle of Fig. 3. The contours of the spanwise velocity component of the modes labeled as $M_{\mathrm{US}_{1}}$ and $M_{\mathrm{US}_{2}}$ are provided in Figs. 7(a) and 7(b), respectively. Such modes are characterized by streamwise-alternated sickle-shaped packets, which are mostly located within the upstream part of the separation bubble. One can notice that the two modes have a similar shape, but the second one is characterized by structures of smaller wavelength. It is noteworthy that in the two-dimensional spectrum computed for the same base flow (see Refs. 10 and 25), these first two families of modes are not recovered. Thus, it is possible to infer that modes $M_{S}, M_{G}, M_{\mathrm{US}_{1}}$, and $M_{\mathrm{US}_{2}}$ are purely threedimensional, since they are not recovered by a twodimensional analysis which does not take into account the spanwise dynamics.

The third family of modes, which is recovered also in the two-dimensional case, is characterized by high-frequency unsteady modes, shown in the third (from left to right) dashed rectangle of Fig. 3. Such eigenvalues are reminiscent of the classical Kelvin-Helmholtz (KH) and TollmienSchlichting (TS) modes predicted by a local approach, ${ }^{21}$ al-
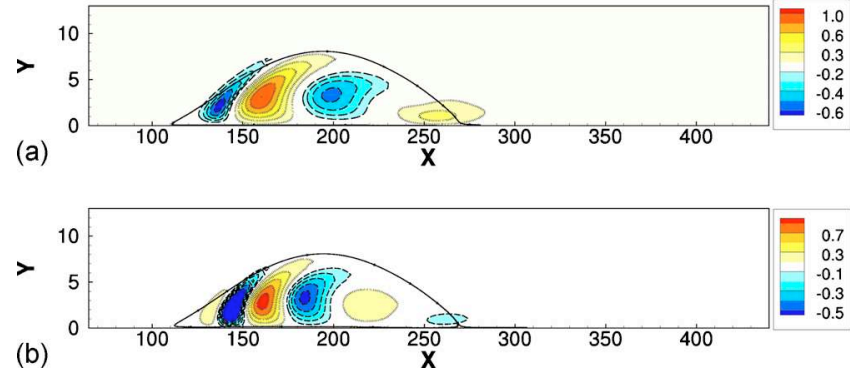

FIG. 7. (Color online) Contours of the spanwise perturbation for the unsteady modes (a) $M_{\mathrm{US}_{1}}$ and (b) $M_{\mathrm{US}_{2}}$ at $\mathrm{Re}=200$. The black line is the separation streamline, whereas dashed contours represent positive perturbations and dotted contours represent negative ones. 


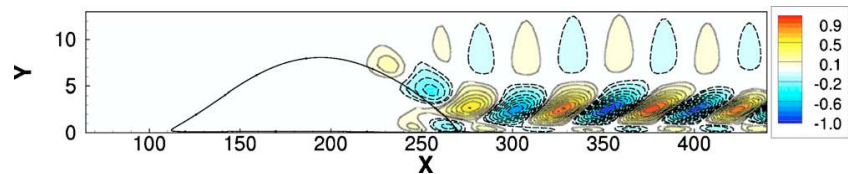

FIG. 8. (Color online) Contours of the streamwise perturbation for mode $M_{\mathrm{TS}_{1}}$ at $\mathrm{Re}=200$. The black line is the separation streamline, whereas dashed contours represent positive perturbations and dotted contours represent negative ones.

though they appear to be slightly tilted in the streamwise direction, as one can observe in Fig. 8, providing the streamwise velocity contours of mode $M_{\mathrm{TS}_{1}}$. The dynamics of such modes, which are the ones responsible for the strong amplifier behavior of the bubble, has been extensively studied in the two-dimensional case for an adverse-pressure induced separation, ${ }^{10,25}$ and for a bubble induced by a step ${ }^{11,12}$ or by a bump. ${ }^{9}$

\section{Analysis of centrifugal modes}

In subsection IV A 1 it has been conjectured that modes $M_{S}$ and $M_{G}$ could both have a centrifugal origin due to their flat roll structure. However, the mechanisms of their generation should differ, since they are characterized by a different distribution of the energy. In particular, $M_{S}$ could be originated by an intrinsic centrifugal instability, as conjectured by previous authors ${ }^{15,16}$ in the case of a geometry-induced separation bubble, whereas the stable mode $M_{G}$ seems to have a convective nature similar to a Gortler instability. Indeed, in previous studies the onset of Gortler vortices has been already observed in the case of the flow past a laminar separation bubble over a flat plate. ${ }^{26}$ In order to verify such conjectures about the origin of modes $M_{S}$ and $M_{G}$, two different analyses are proposed here. Concerning mode $M_{S}$, a generalized form of the Rayleigh criterion $^{27}$ is used, whereas for mode $M_{G}$, a Gortler stability study is performed.

Rayleigh's criterion (see Refs. 28 and 29) is an inviscid condition for centrifugal instability. Such a criterion is based on the physical consideration that in a flow with closed streamlines, instability could be originated if there is an outward decrease in the angular momentum of the base flow. A sufficient condition for instability, based on a generalization of such a criterion to generic closed streamlines, has been provided in Ref. 27 for a two-dimensional inviscid flow. In order to identify the centrifugal zones inside the recirculation region, the Rayleigh discriminant $\Delta(\mathbf{x})$ is defined as follows:

$$
\Delta(\mathbf{x})=2\left(\frac{|\mathbf{U}(\mathbf{x})|}{R(\mathbf{x})}\right) \varpi(\mathbf{x}),
$$

where $\mathbf{x}$ is a point of the considered streamline, $\phi ;|\mathbf{U}(\mathbf{x})|$ is the norm of the base flow velocity; $\varpi(\mathbf{x})=V_{x}-U_{y}$ is the spanwise base flow vorticity; and $R(\mathbf{x})$ is the local radius of curvature of the streamline. The flow is unstable if a closed streamline $\phi_{0}$ exists such that

$$
\max _{\phi_{0}}[\Delta(\mathbf{x})]<0,
$$

where $\max _{\phi}$ denotes the maximum over the streamline $\phi$.

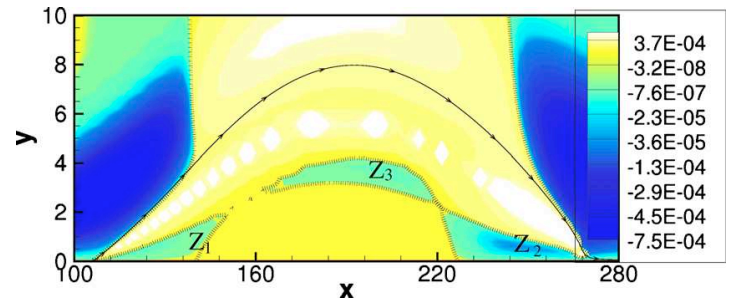

FIG. 9. (Color online) Rayleigh's discriminant contours for the base flow $\mathrm{BF} 1$. The black line is the separation streamline, whereas the dotted line is the zero-discriminant contour. The three centrifugal zones within the bubble are labeled as $Z_{1}, Z_{2}$, and $Z_{3}$.

Rayleigh's discriminant has been computed for the base flow considered here and is shown in Fig. 9. Three centrifugal zones, defined by the negative values of the Rayleigh discriminant, are recovered within the bubble, namely, $Z_{1}$, $Z_{2}$, and $Z_{3}$. Recalling that criterion (9) is only a sufficient condition for instability, it is possible to point out that even if no closed streamline has been recovered along which the Rayleigh discriminant remains negative, the fluid particles which follow closed streamlines within the recirculation zone would periodically pass through these centrifugal zones, so that they could feel the effect of the instability, especially close to the wall where the lowest values of the Rayleigh discriminant are recovered (in $Z_{2}$ ). The large curvature of the streamlines would cause the perturbation to be displaced away from the center of the bubble, where the presence of the wall would force it to move in the spanwise direction, inducing the formation of the roll structures observed in Fig. 5(a).

Rayleigh's discriminant has been computed also for base flows BF2 and BF3, which have the same Reynolds number of BF1 and a blowing and suction wall-normal velocity of smaller intensity (see Fig. 1), and hence have smaller separation bubbles. For smaller bubbles, the spatial extent of the centrifugal zones $Z_{1}, Z_{2}$, and $Z_{3}$ decreases, and the minimum value of $\Delta(x)$ within the bubble is found to increase from $-3.8 \times 10^{-5}$ (for BF1) to $-6.9 \times 10^{-6}$ (for BF3). The amplification rate of mode $M_{S}$ has been computed for all of the base flows, and is provided in Fig. 10 as a function of the minimum value of the Rayleigh discriminant within the bubble. One can observe that the amplification rate decreases when the Rayleigh discriminant increases, indicating that a correlation exists between the intensity of the centrifugal mechanism and the onset of mode $M_{S}$. Finally, it is worth to point out that in Ref. 15, an analytical proof has been given of the centrifugal origin of the three-dimensional primary instability affecting the separated flow over a bump. Indeed, it is

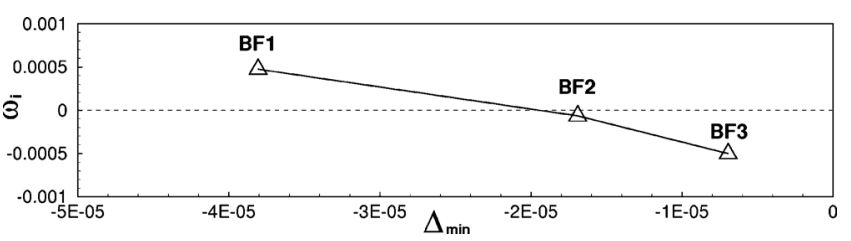

FIG. 10. Amplification rate of mode $M_{S}$ vs the minimum value of the Rayleigh's discriminant for base flows BF1, BF2, and BF3. 

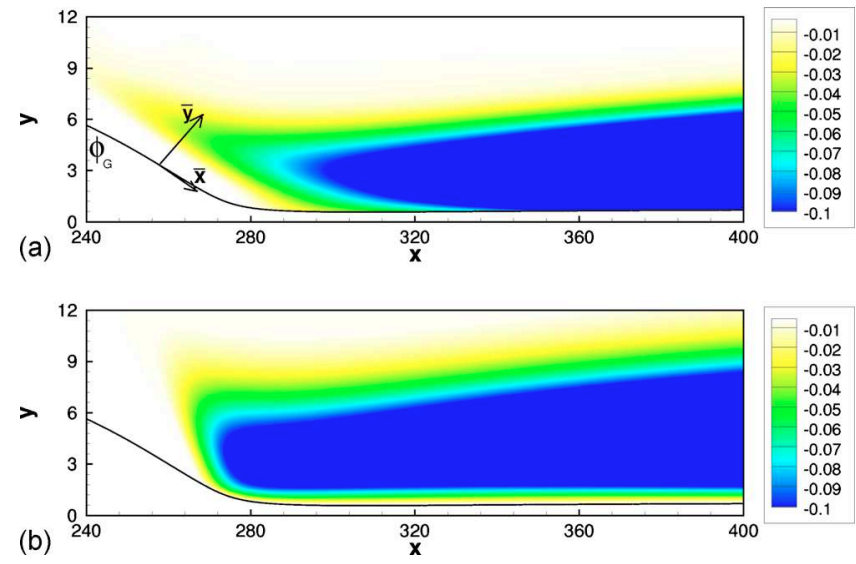

FIG. 11. (Color online) Streamwise perturbation for the global mode $M_{G}$ (a) and for the solution obtained by the space integration of the Gortler equations (b), represented over the streamline $\phi_{G}$.

likely that the same instability mechanism could act in the separated flow considered here, whose centrifugal zones strongly resemble in shape and location the ones described in Ref. 15.

Concerning mode $M_{G}$, a Gortler analysis has been performed for the streamlines past the bubble. At this purpose, the formalism of Ref. 30 has been followed, which proposed to study the influence of the curvature of the wall on the instability dynamics of a three-dimensional boundary layer. It is straightforward that in the case under consideration, no curved wall is present, but it has been conjectured that the curvature of the streamlines past the separation bubble could have a similar effect on the instability of the boundary layer. ${ }^{31}$ Thus, a local cylindrical coordinate system $(\bar{x} ; \bar{y} ; \bar{z})$ has been defined along a streamline past the separated zone $\phi_{G}$ [see Fig. 11(a)], having its origin at $x_{0_{G}}=198, y_{0_{G}}=8.4$, namely,

$$
\left\{\begin{array}{l}
\bar{x}=\Theta R \\
\bar{y}=(r-R) \sqrt{\mathrm{Re}} \\
\bar{z}=z \sqrt{\mathrm{Re}},
\end{array}\right.
$$

where $R$ is the local radius of curvature, $\Theta$ is the azimuthal coordinate, and $r$ is the radial one. The Gortler equations have been recovered in this local cylindrical coordinate system (see Appendix B) and have been integrated for several values of $\beta$. Figure 11(b) shows the streamwise component of the perturbation for $\beta=0.17$. Such a perturbation has been found very similar to the global mode $M_{G}$, which is provided in Fig. 11(a) for $\beta=0.17$ for comparison. The contours of the spanwise vorticity perturbation provided in Fig. 12 show that the perturbation obtained by the Gortler model presents the same flat roll structure of the global mode $M_{G}$, indicating that the mechanism underlying the onset of such longitudinal vortex is of the Gortler type. As a further validation, the spatial amplification of the perturbation has been analyzed by computing the energy at the azimuthal position $\bar{x}$,
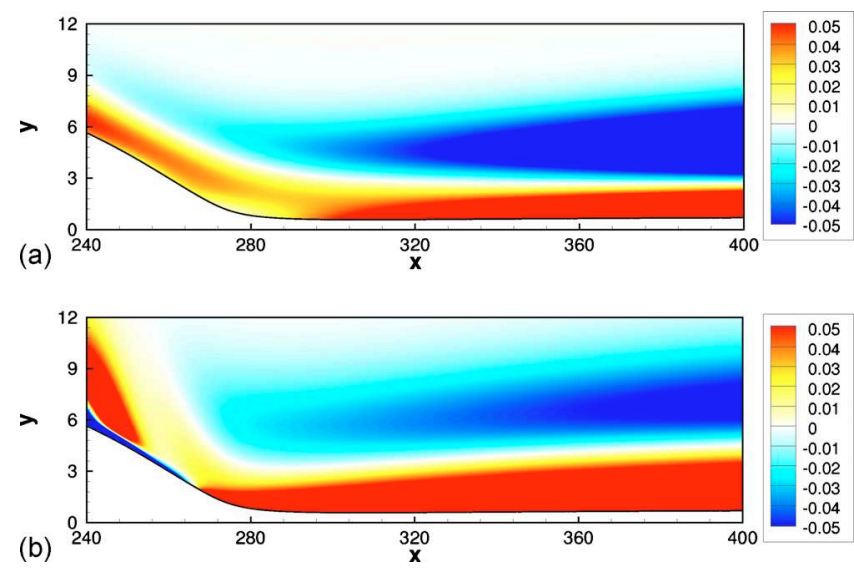

FIG. 12. (Color online) Spanwise vorticity of the perturbation for the global mode $M_{G}$ (a) and for the solution obtained by the space integration of the Gortler equations (b), represented over the streamline $\phi_{G}$.

$$
E_{x}(\bar{x})=\int_{0}^{\bar{L}_{z}} \int_{0}^{\bar{y}_{\max }}\left(\bar{u}^{2}+\bar{v}^{2}+\bar{w}^{2}\right) d \bar{y} d \bar{z}
$$

along the streamline $\phi_{G}$. For both the Gortler approximation and the global analysis, the largest value of the spatial energy gain $E_{x}(\bar{x}) / E_{x}\left(\bar{x}_{0}\right)$ is recovered slightly downstream of the reattachment point, close to the point where the maximum value of $G$ is attained, reaching a value close to 5.6 (see Fig. 13). Moreover, one can observe that both solutions present a positive slope at the outlet of the domain, namely, $\partial E_{x}(\bar{x}) / \partial \bar{x} \approx 0.0345$ for the Gortler approximation and $\partial E_{x}(\bar{x}) / \partial \bar{x} \approx 0.0234$ for the global perturbation with $\beta=0.17$. Such a slight discrepancy in the slope value could be ascribed to some differences between the two approaches. In fact, in the global one, the spatial amplification should be corrected by the temporal damping in order to be comparable to the one obtained by the space-marching technique, which neglects the time evolution of the perturbation. ${ }^{32}$ In both cases, the spatial amplification of the energy in the attached boundary layer induces the generation of the streamwise vortices shown in Figs. 11 and 12. The maximum value of such an amplification has been measured at $\beta=0.19$ for the spatially developing perturbation computed using the Gortler approximation. A comparison with the result of the global eigenvalue analysis would be made in subsection IV A 3.

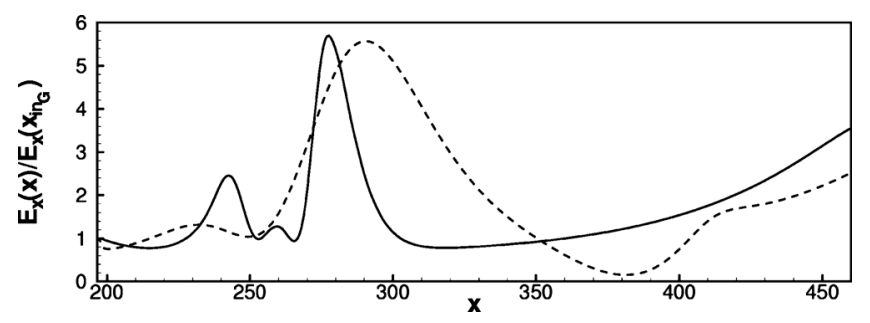

FIG. 13. Streamwise distribution of the energy $E_{x}$ for the perturbation resulting from the space integration of the Gortler equations (dashed line) and for the global mode $M_{G}$ (solid line). The energy has been plotted with respect to the $x$ variable to allow a comparison with Figs. 11 and 12 . 


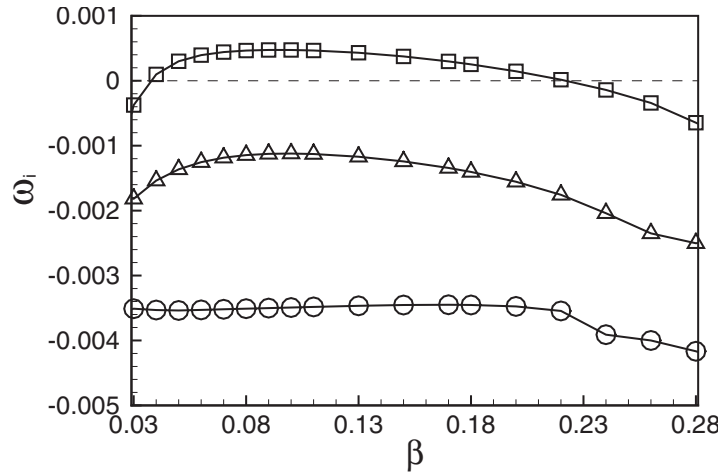

FIG. 14. Amplification rate for the unstable mode $M_{S}$ (squares), unsteady mode $M_{\mathrm{US}_{1}}$ (triangles), and Gortler mode $M_{G}$ (circles) vs the spanwise wavenumber $\beta$.

\section{Spanwise analysis}

The influence of the spanwise wavenumber $\beta$ on the amplification rate of modes $M_{S}, M_{\mathrm{US}}$, and $M_{G}$ is investigated here. Several spectra have been computed for the base flow BF1 and different values of $\beta$. Figure 14 shows the curves of the amplification rate $\omega_{i}$ versus the spanwise wavenumber $\beta$ for modes $M_{S}$ (squares), $M_{\mathrm{US}_{1}}$ (triangles), and $M_{G}$ (circles), respectively. The amplification rate of the modes is shown only for $0.03 \leq \beta \leq 0.28$ due to the fact that the steady threedimensional modes change their nature becoming a complex pair of unsteady modes when the spanwise length of the domains becomes too narrow or too large. ${ }^{16}$ Figure 14 shows that mode $M_{S}$ is unstable for $0.038 \leq \beta \leq 0.22$. Such a mode reaches the maximum amplification rate for $\beta=0.1$ (hereafter called the most amplified spanwise wavenumber for mode $\left.M_{S}, \beta_{S_{\max }}\right)$. It is worth to point out that $\beta_{S_{\max }}$ scales with the size of the bubble, so it seems to be an intrinsic property of the separation bubble. The amplification rate of mode $M_{\mathrm{US}_{1}}$ achieves its maximum for the same value of $\beta$ but, unlike mode $M_{S}$, such mode is always stable. Finally, mode $M_{G}$ presents an higher value of the most amplified spanwise wavenumber, namely, $\beta_{G_{\max }}=0.17$. Such a discrepancy could be a consequence of the different nature of the two steady modes, the first one $\left(M_{S}\right)$ having an intrinsic origin, the second one $\left(M_{G}\right)$ arising from a convective mechanism. Indeed, it is worth to recall that the peak of the spatial amplification of the perturbation computed with the Gortler approximation was obtained for $\beta=0.19$, which is very close to $\beta_{G_{\max }}$. This is a further validation of the Gortler convective nature of mode $M_{G}$. Concerning the amplification rate of the Tollmien-Schlichting modes (not shown), it decreases with $\beta$ as expected.

\section{Transient analysis}

The transient amplification of the perturbations is analyzed here. Figure 15 provides the optimal energy-gain curves computed by the global model for several values of $\beta$. The energy gain peak, which reaches a value of about $3 \times 10^{8}$ in the two-dimensional case (see the solid line in Fig. 15 ), has been found to decrease with $\beta$, as shown by the dashed, dotted, and dashed-dotted lines corresponding to $\beta=0.1, \beta=0.2$, and $\beta=0.3$, respectively. Figure 16 shows the

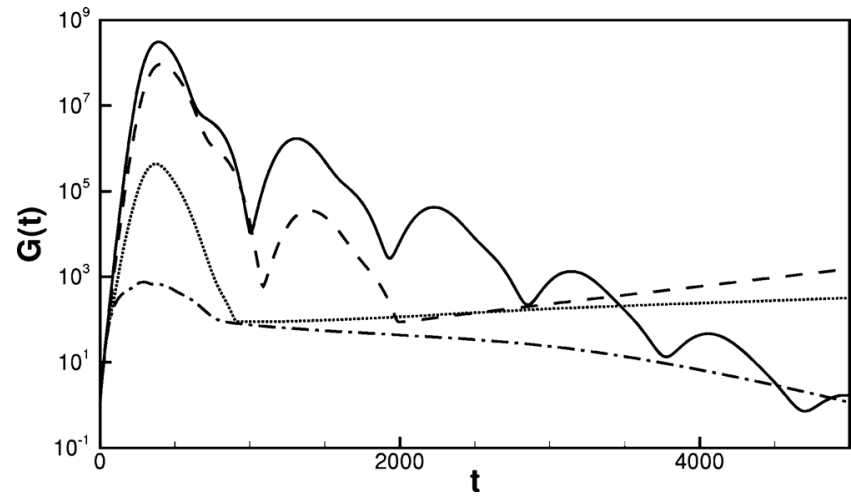

FIG. 15. Optimal energy gain curves obtained by the global model for $\beta=0$ (solid line), $\beta=0.1$ (dashed line), $\beta=0.2$ (dotted line), and $\beta=0.3$ (dashed-dotted line).

optimal initial perturbation at time $t=0$ and its linear evolution at optimal time $T_{\max }=400$ for $\beta=0$ [frames (a) and (b), respectively] and at $T_{\max }=410$ for $\beta=0.1$ [frames (c) and (d), respectively]. One can observe that the optimal perturbations at $\beta=0$ and $\beta=0.1$ are both characterized at $t=0$ by a wave packet localized close to the separation point which amplifies itself along the separation streamline turning into TS waves at $t=T_{\max }$. Such optimal perturbations present similar shape and structure, although the one with $\beta=0.1$ is tilted in the streamwise direction.

It is worth to notice in Fig. 15 the onset of the asymptotically unstable steady mode $M_{S}$ for the curves with $\beta=0.1$ and $\beta=0.2$, the second one showing a lower amplification rate. One could also observe for $\beta=0$ and $\beta=0.1$ the presence of a modulation of the energy gain curves. Such a
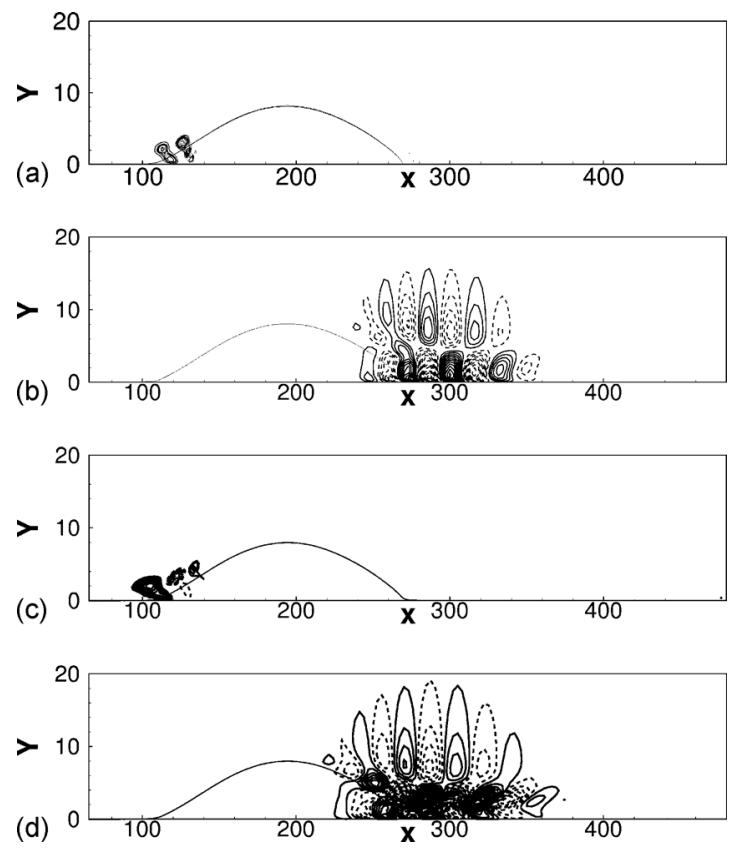

FIG. 16. Contours of the streamwise perturbation computed by the global model: optimal two-dimensional perturbation at (a) $t=0$ and (b) $t=T_{\max }=400$; optimal three-dimensional perturbation with $\beta=0.1$ at (c) $t=0$ and (d) $t=T_{\max }=410$. The solid lines indicate positive values, whereas the dashed lines represent negative ones. 


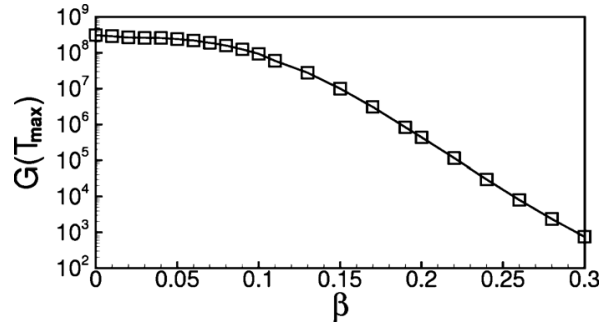

FIG. 17. Peak value of the optimal energy gain obtained by the global model vs the spanwise wavenumber $\beta$.

phenomenon is known as "flapping," which has been extensively studied in the two-dimensional framework for separated flows. ${ }^{9,10}$ It is originated by the interaction of the most unstable modes of the spectrum, which are advected back and forward in the streamwise direction due to the recirculation inside the bubble. It is noteworthy that the flapping phenomenon is not recovered for high values of $\beta$ due to the lowering of the KH/TS branch of the spectrum for increasing spanwise wavenumber. In Fig. 17 the dependence of the maximum value of the optimal energy gain on the spanwise wavenumber $\beta$ is shown. Although such a parameter decreases with $\beta$, the decrease has been found to be very slow for $\beta<0.1$. Such a behavior could be due to the coexistence of the convective amplification of $\mathrm{KH} / \mathrm{TS}$ waves, lift-up mechanism, and energy growth due to the asymptotic instability of mode $M_{S}$. In particular, for a large separation bubble, like the one considered here, the energy gain is mostly due to the amplification of KH/TS waves, which reaches the highest value of spatial amplification when $\beta=0 .{ }^{21}$ On the other hand, the amplification due to the lift-up mechanism, which is active only when $\beta \neq 0$ and increases with the spanwise wavenumber up to $\beta=0.6$, is able to reach an energy gain of order $10^{2}$ for an attached boundary layer at the same Reynolds number. ${ }^{33-35}$ An even lower amplification is reached at short time by the weakly growing unstable mode $M_{S}$, which provides at $t \approx 400$ (close to the optimal time $\left.T_{\max }\right)$ a value of $G(t)$ of order 10. Such amplification values are very low with respect to the one due to KH/TS waves, so that an optimal dynamics is recovered for the $\mathrm{KH} / \mathrm{TS}$ optimal wavenumber, $\beta=0$. It is worth to notice that for a separation bubble in which the two-dimensional amplification reaches a maximum value of the energy gain comparable to the one due to the three-dimensional mechanisms, an optimal wavenumber greater than zero would be recovered (see the results in Refs. 11 and 12 for geometry-induced separation bubbles).

In section IV $\mathrm{B}$, the dynamics of the global modes discussed here will be studied in the nonlinear framework using direct numerical simulations.

\section{B. Dynamics of small amplitude perturbations: The onset of centrifugal global modes}

\section{Two-dimensional perturbations}

This section aims at verifying the existence in a nonlinear framework of the modes previously identified by the global eigenvalue analysis and at investigating their role in the

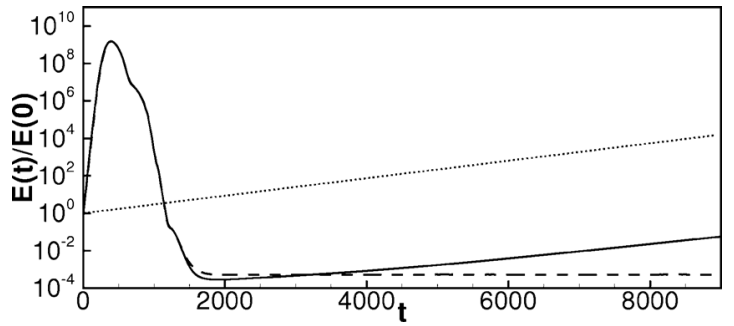

FIG. 18. Time evolution of the normalized energy computed by a threedimensional DNS (solid line) and a two-dimensional one (dashed line) initialized with the optimal perturbation obtained at $\mathrm{Re}=200$ with amplitude $A_{0}=10^{-8}$. The dotted line represents the theoretical slope of the amplification rate of mode $M_{S}$ obtained by the global model.

transition mechanism. Nonlinear simulations have been performed initializing the computation by superposing to the base flow the two-dimensional optimal perturbation with amplitude $A_{0}=10^{-8}$. The length of the domain in the spanwise direction has been chosen equal to the most amplified wavelength of mode $M_{S}\left(L_{z}=2 \pi / \beta_{S_{\max }}=62.8\right)$. Figure 18 shows the time evolution of the normalized energy obtained by the three-dimensional DNS (solid line) and by a twodimensional one (dashed line) performed with the same parameters. It is worth to notice that for $t<1500$, the two energy-gain curves are overlapped, meaning that the amplification dynamics of the perturbation is quasi-twodimensional. In both cases the disturbance initially placed close to the separation point is amplified and convected downstream, resulting in a packet of TS waves in the attached zone. ${ }^{10}$ Some differences are recovered in the asymptotic regime due to the appearance of the threedimensional steady weakly growing mode which has been found to be unstable at $\mathrm{Re}=200$. Indeed, looking at the energy gain curve, one can observe that after the transient has passed, the perturbation begins to be amplified with an exponential growth rate. By computing the growth rate parameter,

$$
\sigma_{i}(T)=\left.\frac{1}{2 E(T)} \frac{d E(t)}{d t}\right|_{T},
$$

at a large time $T$, one obtains $\sigma_{i}=0.000463$, which is very close to the one recovered by the global model for mode $M_{S}$, $\omega_{i}=0.000474$ (the dotted line in Fig. 18 shows the growth of the energy theoretically associated with such an amplification rate).

The perturbation has been extracted from the DNS at several times. Figure 19 shows the spanwise vorticity perturbation isosurfaces at a very large time, $t=12000$. Vorticity rolls, very similar to the ones typical of mode $M_{S}$, can be observed, confirming that the flow perturbed by a small amplitude disturbance tends toward the linearly most amplified three-dimensional mode asymptotically. Nevertheless, at small times, the dynamics is more complex, as it can be observed in Fig. 20, providing the spanwise velocity contours in the plane at $z=26$. Figure 20(a) shows at $t=600$ the contours of the spanwise component of the $\mathrm{KH}$ and $\mathrm{TS}$ waves being convected downstream by the mean flow. At this time the dynamics is almost two-dimensional, so that the 


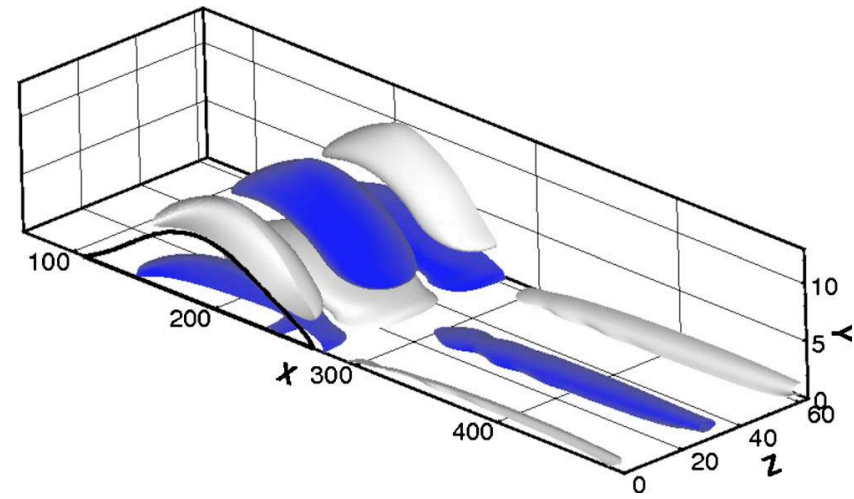

FIG. 19. (Color online) Isosurfaces of the spanwise vorticity of the perturbation extracted at $t=12000$ from the DNS with $L_{z}=62.8$ initialized by the two-dimensional optimal disturbance with amplitude $A_{0}=10^{-8}$. The solid line is the separation streamline, whereas dark and light surfaces represent the 0.15 and -0.15 values, respectively. The perturbation has been normalized by its maximum value.

spanwise component of the velocity is very small with respect to the wall-normal and streamwise ones. Nevertheless, the dynamics begins to differ qualitatively from the purely two-dimensional one when a small amount of perturbation is "left behind" in the separated zone close to the reattachment point. Such a spanwise perturbation is convected upstream by the recirculating base flow, increasing its extension due to the strong nonparallel effects which are present in this region [see Fig. 20(b)]. Therefore, when the KH/TS wave packet leaves the separated zone, a residual perturbation is present inside the bubble, which is characterized by two sickleshaped packets with alternating sign, as shown in Fig. 20(c). The shape of such perturbations could be due to the presence of the negative shear close to the wall, which stretches the perturbation upstream, and of the positive shear close to the separation streamline, stretching the perturbation downstream. Moreover, it is worth to point out that the shape of such wave packets recalls the one of the unsteady global mode $M_{\mathrm{US}}$ provided in Fig. 7(a). The further evolution of the spanwise perturbation in Fig. 20(d) shows that the main part of the wave packet continues to be convected upstream, whereas a residual part remains close to the reattachment point, although being stretched upstream. It is noteworthy that this part of the perturbation is placed in the strong centrifugal zone $Z_{2}$ (shown in Fig. 9); therefore, it is likely that such a spanwise perturbation increases in size under the effect of the outward decrease of the momentum close to the wall. At $t=2100$ [see Fig. 20(e)], the wave packet placed upstream has decreased its size in the wall-normal direction and has approached the separation point, whereas the downstream part of the perturbation continues to be stretched in the streamwise direction, increasing in size. Again, it is worth to point out that the upstream part of the perturbation is very similar to the unsteady global mode $M_{\mathrm{US}_{2}}$ provided in Fig. 7(b). Finally, one can observe in Fig. 20(f) that at $t$ $=2900$ the upstream negative spanwise perturbation has been stretched along the first half of the separation streamline, so that it takes the form of the unstable three-dimensional mode $M_{S}$ [see Fig. 4(b)].

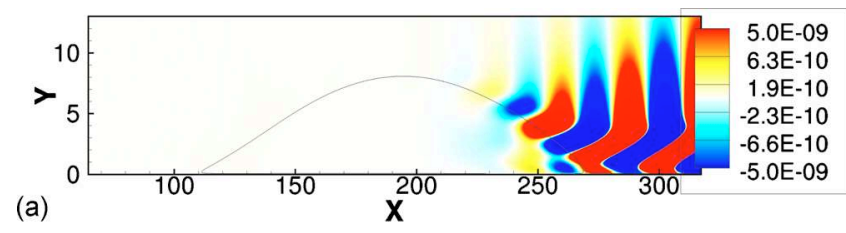

(b)

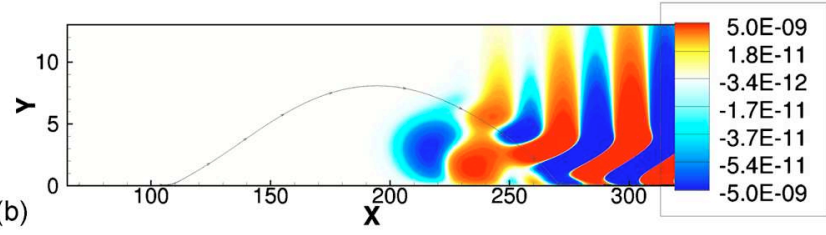

(c)
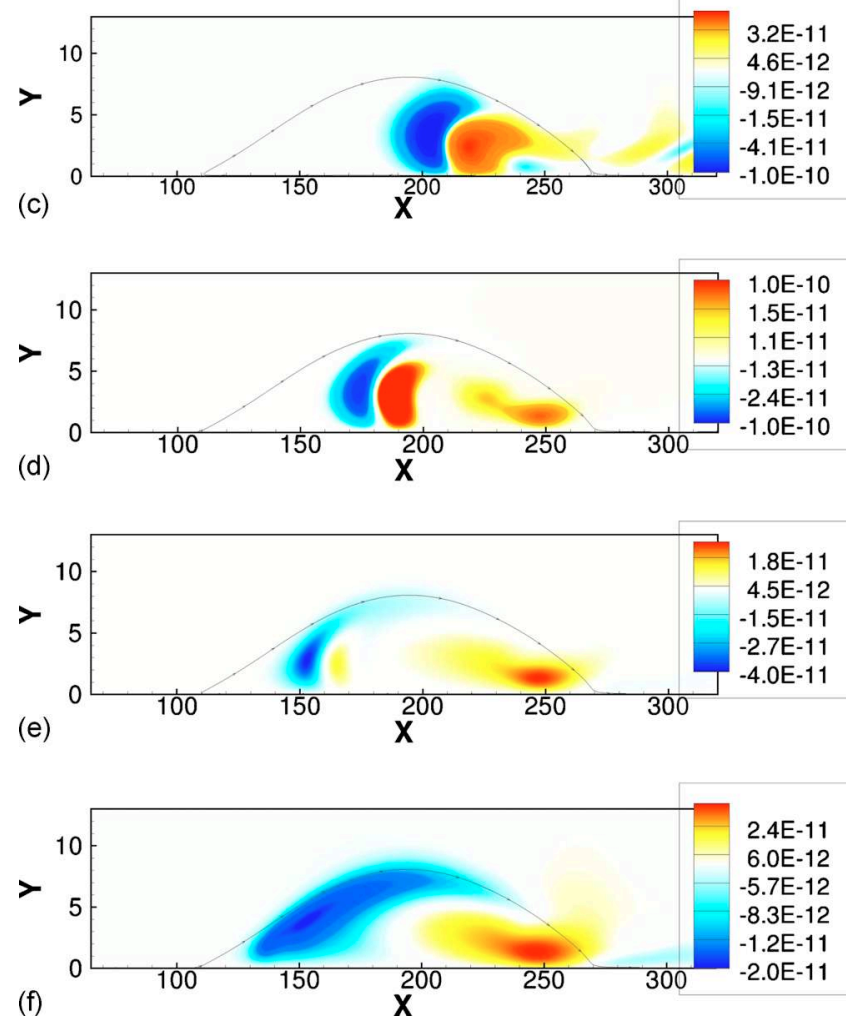

FIG. 20. (Color online) Contours of the spanwise perturbation in the separated region on the $z=26$ plane at time instants: (a) $t=600$, (b) $t=700$, (c) $t=900$, (d) $t=1500$, (e) $t=2100$, and (f) $t=2900$. The solid line is the separation streamline.

Therefore, it has been found that several global modes appear in the $x-y$ plane during the changeover of the early transient toward the asymptotic dynamics. The time evolution of the spanwise shape of the perturbation is now investigated. Indeed, since the spanwise length of the domain is the one giving the largest amplification rate of the unstable global mode $M_{S}$ (being $L_{z}=2 \pi / \beta_{S_{\max }}$ ), one could infer that the dominant spanwise frequency would be equal to $\beta_{S_{\max }}$ when nonlinear effects are weak. In order to verify this, the Fourier transform in the spanwise direction of the streamwise component of the perturbation has been performed at several instants of time at the streamwise and wall-normal position within the separated region where the perturbation of highest amplitude is localized. Figures 21(a) and 21(b) show a wide spectrum of frequencies at short times $(t=700$ and 1700 , respectively) due to the interactions of the several 

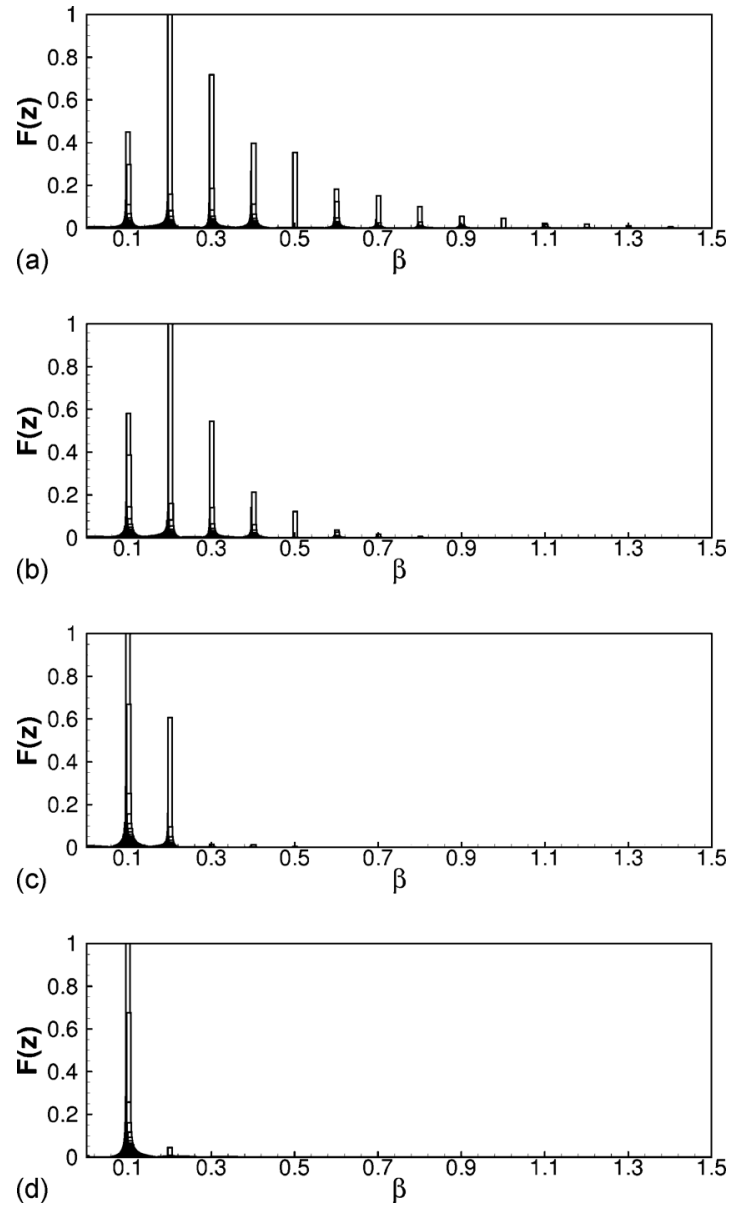

FIG. 21. Fourier transform in $z$ at the point of maximum amplification within the separated region of the streamwise perturbation; extracted from the DNS initialized by the two-dimensional optimal initial perturbation, at the time instants: (a) $t=700$, (b) $t=1700$, (c) $t=2500$, and (d) $t=12000$.

modes affecting the dynamics of the flow, as previously shown. At larger times [see Figs. 21(c) and 21(d) for $t=2500$ and 12000 , respectively], the spectrum results to be narrower, and dominated by the most amplified wavenumber $\beta_{S_{\max }}$, since the asymptotic dynamics is dominated by the occurrence of the unstable mode $M_{S}$. Most importantly, one can notice in Figs. 21(a) and 21(b) that for $t<2500$ the dominant frequency is not $\beta_{S_{\max }}$ but its double, $\beta=0.2$. Such a behavior could not be ascribed to nonlinear effects or secondary instability of the growing waves due to the very low amplitude of the perturbations at $t=1700$ (see the energy level at such time in Fig. 18). Such a result means that at small times the spanwise perturbation is essentially composed by packets having a spanwise wavelength smaller than the most amplified one, which slowly increase their size in time, approaching the spanwise wavenumber $\beta_{S_{\max }}$. A similar behavior is observed when the optimal perturbation is initialized with a larger amplitude, $A_{0}=10^{-6}$. Moreover, Fig. 22 shows that the flow is characterized by elongated structures which closely recall mode $M_{G}$. Thus, it is likely that such a mode dominates the dynamics during the transient inducing in the frequency spectrum a dominating wavenumber close to 0.17 . Indeed, although $M_{G}$ is asymptotically stable, it could be excited during the transient by means of a sensitiv-

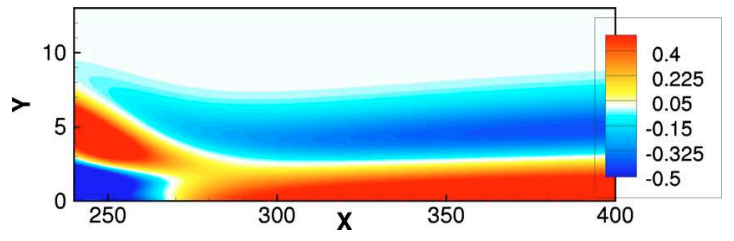

FIG. 22. (Color online) Blow-up of the shaded contours of the spanwise vorticity of the perturbation extracted from the DNS initialized by the optimal initial perturbation with amplitude $A_{0}=10^{-6}$ at $z=42$ and $t=2500$. The perturbation has been normalized by its maximum value.

ity mechanism which will be discussed in the following. Nevertheless, due to the asymptotic instability of mode $M_{S}$, at a larger time the structures having smaller wavelength begin to merge, resulting in rolls of spanwise vorticity alternated at the most amplified wavenumber $\beta_{S_{\max }}$, so that mode $M_{S}$ becomes the dominant one.

In order to verify such a hypothesis on the excitation of the Gortler mode, a DNS has been performed in which the spanwise domain length has been chosen equal to the most amplified wavelength for the onset of the Gortler mode $\left(L_{z}=2 \pi / \beta_{G_{\max }}=37\right)$. In order to perform a comparison with the results shown in Fig. 21, an initial perturbation identical in shape and amplitude to the one previously used has been superposed to the base flow. Fourier transforms in $z$ of the streamwise velocity signal have been performed at the point of greater amplification within the separated region at $t=700$ and 1700. As shown in Fig. 23, the dominant frequency is again coincident with the Gortler most amplified wavenumber $\left(\beta=\beta_{G_{\max }}=0.17\right)$. It is worth to notice that in the previous case (for $L_{z}=2 \pi / \beta_{S_{\max }}=62.8$ ) the dominant wavenumber was the double of the minimum one allowed in the flow $(\beta=0.1)$, whereas for $L_{z}=2 \pi / \beta_{G_{\max }}=37$ it is equal to such minimum, which is $\beta=0.17$. Thus, in both cases the wavenumber that dominates the flow during the transient is very close to the most amplified wavenumber for the onset of the Gortler mode, corroborating the hypothesis that such
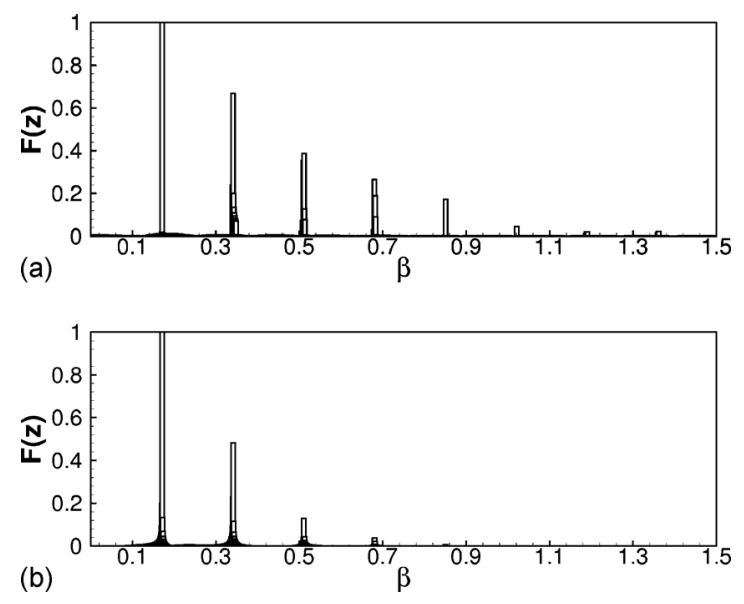

FIG. 23. Fourier transform in $z$ at the point of maximum amplification within the separated region of the streamwise perturbation; extracted from the DNS with $L_{z}=2 \pi / \beta_{G_{\max }}=37$ initialized by the two-dimensional optimal initial perturbation, at the time instants (a) $t=700$ and (b) $t=1700$. 

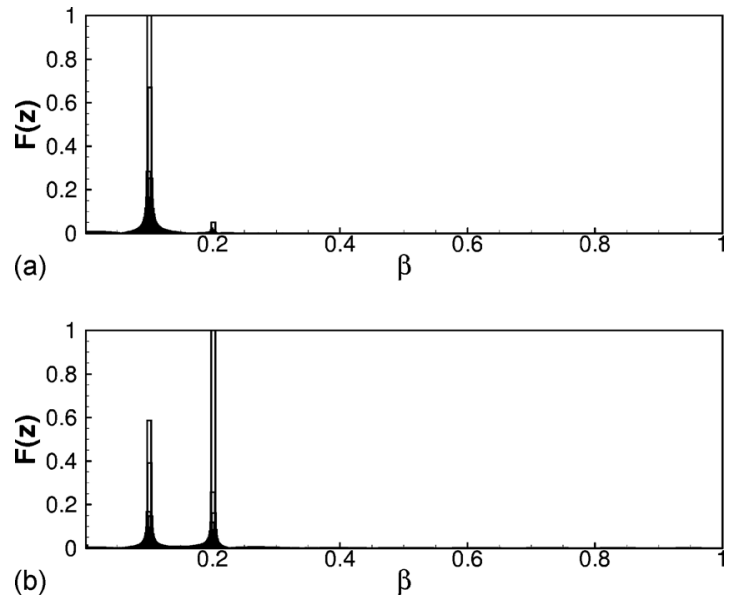

FIG. 24. Fourier transform in $z$ at the point of maximum amplification within the separated region of the streamwise perturbation; extracted from the DNS with $L_{z}=2 \pi / \beta_{S_{\max }}=62.8$ initialized by the optimal initial perturbation, for $\beta=0.1$ at the time instants (a) $t=700$ and (b) $t=4700$.

mode has indeed a role in the transient process leading to the asymptotic self-sustained amplification of mode $M_{S}$.

\section{Three-dimensional perturbations}

The dynamics of the flow has been analyzed when it is perturbed with three-dimensional perturbations of small amplitude. The optimal spanwise-modulated perturbation computed by the global model for $\beta_{S_{\max }}=0.1$ has been superposed to the base flow with an amplitude $A_{0}=10^{-8}$. The transient evolution of the perturbation does not present remarkable differences from the one observed in the previous simulations, except for the spanwise-modulation of the TS waves convected by the separation bubble, and the lower peak value of the energy gain curve. The Fourier transform in the spanwise direction of the streamwise component of the velocity at the point of maximum amplification within the bubble is shown for $t=700$ and 4700 in Figs. 24(a) and 24(b), respectively. In the early transient phase preceding the onset of mode $M_{S}$, the dominant spanwise wavenumber remains the one at which the flow has been perturbed, which is equal to the most amplified wavenumber for mode $M_{S}$. Surprisingly, at slightly larger times (approximately for $1000<t<6000$ ), the dominant wavenumber doubles, as shown in Fig. 24(b) for $t=4700$. Such a behavior could not be ascribed to nonlinear effects or secondary instability of the growing waves due to the very low amplitude of the perturbations at this time. In fact, considering that an initial perturbation of magnitude $A_{0}=10^{-8}$ has been used to initialize the DNS, and that at $t=4700$ an amplification of about 6.8 has been reached, one could conclude that at such times nonlinearity does not play any role. The same conclusion can be drawn about secondary instability, which is usually triggered when the perturbations reach a finite amplitude. Thus, as in the previous case, this behavior has to be ascribed to some linear mechanism. Indeed, the dominating wavenumber, which differs from the initial one, is very close to $\beta_{G_{\max }}$; such a wavenumber dominates the transient dynamics leading to the formation of streamwise vortices recalling mode

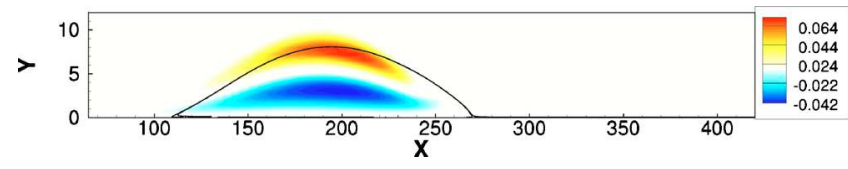

FIG. 25. (Color online) Contours of the optimal spanwise perturbation computed by the global model for $T=5000$ and $\beta=0.1$.

$M_{G}$, as in the previously discussed case. Such results confirm the important role of the Gortler mode in the self-sustained process leading the flow to asymptotic instability, being able to arise in the flow even when the wavenumber of the initial perturbation is equal to $\beta_{S_{\max }}$. The same dynamics is observed in simulations initialized by the same perturbation at a larger amplitude, $A_{0}=10^{-6}$, although some differences due to nonlinear effects are observed.

On the other hand, a different behavior is recovered when the base flow is initialized with the optimal asymptotic initial condition estimated by the global model at a large time, namely, $t=5000$ for $\beta=0.1$, shown in Fig. 25. In fact, the Fourier spectra computed in the spanwise direction at different times are always dominated only by $\beta_{S_{\max }}$. Thus, it could be inferred that the onset of the Gortler modes depends on the spatial structure of the perturbation. In particular, the mechanism of selection of modes $M_{S}$ and $M_{G}$ could be linked to some intrinsic features of the flow, such as its structural sensitivity. In Ref. 18, it is discussed how finite amplitude perturbations present into the flow could induce modifications of the Navier-Stokes operator; every mode could thus be differently affected by such modifications, depending on its zones of maximum structural sensitivity. The sensitivity of an eigenvalue $\omega$ to a perturbation of the operator $\delta \mathbf{A}$ is given by ${ }^{18}$

$$
\delta \omega=\varepsilon \frac{\left(\hat{\mathbf{q}}^{\dagger}, \delta \mathbf{A} \cdot \hat{\mathbf{q}}\right)}{\left(\hat{\mathbf{q}}^{\dagger}, \mathbf{B} \cdot \hat{\mathbf{q}}\right)},
$$

where $\hat{\mathbf{q}}$ is the eigenvector associated with $\omega$, and $\hat{\mathbf{q}}^{\dagger}$ is its adjoint counterpart. In Ref. 18, the authors demonstrate that $\delta \omega$ is always smaller than

$$
\lambda(x, y)=\frac{\|\hat{\mathbf{q}}\|\left\|\hat{\mathbf{q}}^{\dagger}\right\|}{\int_{x_{\text {in }}}^{x_{\text {out }}} \int_{0}^{L_{y}} \hat{\mathbf{q}} \cdot \hat{\mathbf{q}}^{\dagger} d x d y},
$$

where $\|\cdot\|$ indicates the modulus of the vector. Thus, the sensitivity vanishes in regions where either the direct global mode or the adjoint global mode vanishes, whereas the regions where $\lambda$ is greater than zero may be identified as the wave-maker regions. Such regions have been identified for both modes $M_{S}$ and $M_{G}$ and are provided in Figs. 26(a) and 26(b), respectively. One could observe that the sensitivity region associated with mode $M_{S}$ extends deeply inside the recirculation bubble, whereas for mode $M_{G}$, it is localized in the shear layer. Thus, it is possible to conclude that $\mathrm{KH}$ waves being convected through the shear layer on the bubble, like the ones generated by the optimal initial perturbation, would excite the $M_{G}$ mode more effectively than a perturbation whose energy is distributed over all the recirculation zones, like the one in Fig. 25. Such a result could mean that in the linear regime a mechanism exists for the 


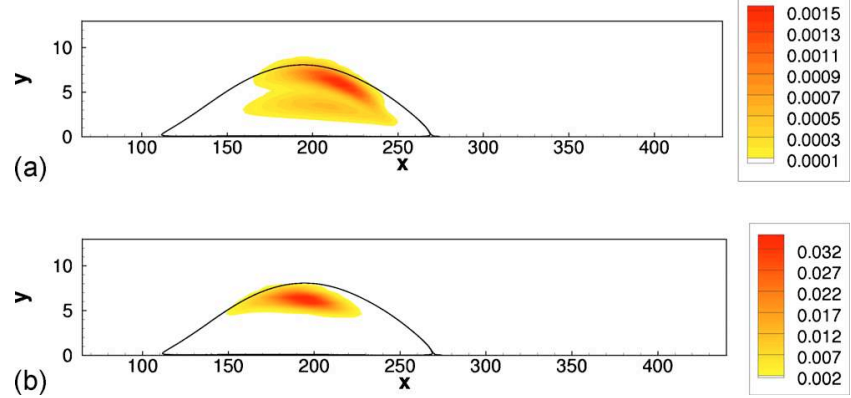

FIG. 26. (Color online) Contours of the $\lambda$ parameter estimating the structural sensitivity of modes (a) $M_{S}$ and (b) $M_{G}$.

onset of mode $M_{S}$, which does not depend from the excitement of the Gortler mode, but that usually such a mechanism is skipped because it is not the one leading to the largest energy amplification, and mode $M_{G}$ is often observed during the transient leading to mode $M_{S}$. Therefore, a perturbation placed at the upstream part of the bubble leads to an energy amplification through the $\mathrm{KH}$ mechanism; transiently evolves in the form of TS waves and unsteady wave packets recalling modes $M_{\mathrm{US}}$; consequently excites the Gortler modes and finally takes the form of mode $M_{S}$.

The conjecture discussed here on the role of mode $M_{G}$ will be further analyzed in section IV C, dealing with the dynamics of large amplitude perturbations.

\section{Dynamics of large amplitude perturbations: Primary and secondary transitions}

Figure 27 shows the energy gain provided by a DNS initialized by the two-dimensional optimal disturbance with an amplitude $A_{0}=10^{-4}$. As one can observe, two zones of transition are identified (enclosed by the dashed lines, hereafter referred to as primary and secondary transitions). The first one for $400<t<1000$ is transient and is due to the strong spatial amplification of KH/TS waves. For $t>1000 \mathrm{a}$ relaminarization occurs, followed by the onset of mode $M_{S}$. At $t>8000$, a self-sustained transition occurs, which is due to the presence of the asymptotically unstable mode $M_{S}$, although at $t \approx 10000, t \approx 11500, t \approx 13500$, and $t \approx 19500$, the perturbation is found to decrease temporarily, affecting

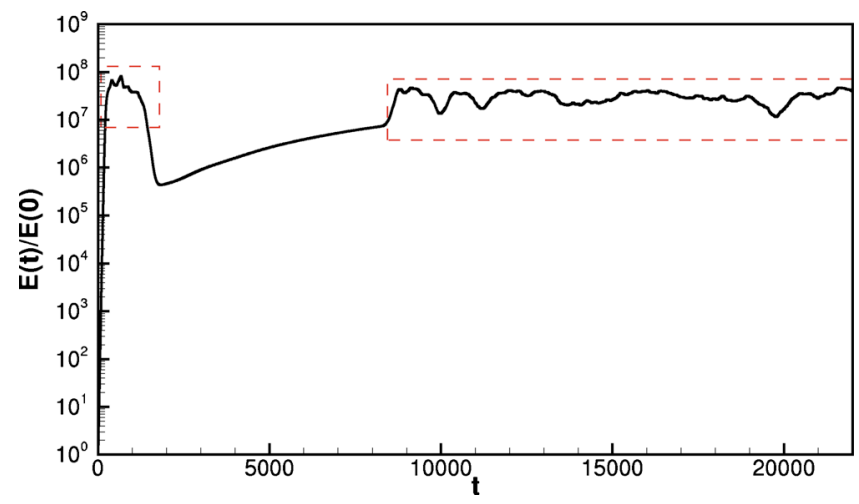

FIG. 27. (Color online) Energy gain obtained by a DNS initialized by the optimal perturbation with amplitude $A_{0}=10^{-4}$.
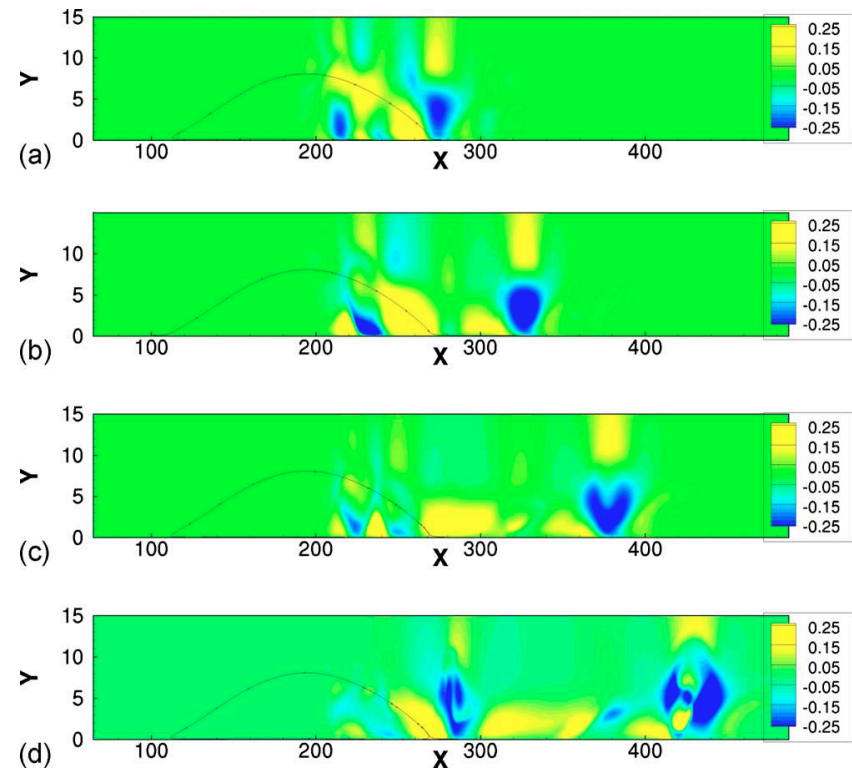

FIG. 28. (Color online) Contours of the streamwise perturbation on the plane $z=25$ extracted from the DNS initialized by the optimal perturbation with amplitude $A_{0}=10^{-4}$ at (a) $t=300$, (b) $t=400$, (c) $t=500$, and (d) $t=600$.

the energy gain curve with a low-frequency modulation. In the following, the dynamics of the perturbation for $400<t$ $<1000$ and for $t>8000$ will be analyzed in detail.

\section{K-type transition}

Figures 28(a)-28(d) show the streamwise velocity contours at $t=300,400,500$, and 600, respectively. At $t=300$, the dynamics is similar to the one recovered by a twodimensional computation in the case of a large-amplitude initial perturbation. ${ }^{10}$ A convectively amplified twodimensional wave packet is identified in the attached zone, which is the result of the excitement, by means of the high sensitivity of the base flow, of a local spatial instability. ${ }^{10}$ As shown in Fig. 28(b), such a wave packet is convected downstream and is amplified until nonlinear interactions kick in, introducing a modulation in the spanwise direction. Some subharmonics of the dominant wavelength are recovered also in the streamwise direction [see Fig. 28(c) at $x=380$ ]. At the same time, another wave packet is created and shed by the bubble, as shown in Fig. 28(d), which undergoes an irregular evolution. Therefore, an unsteady behavior is established in the attached zone, which highly resembles the scenario of the $K$-type transition. ${ }^{36}$ In particular, regions of high wall-normal shear can be identified, which are associated with strong velocity spikes, shown in Fig. 29 by the isolines of the span-

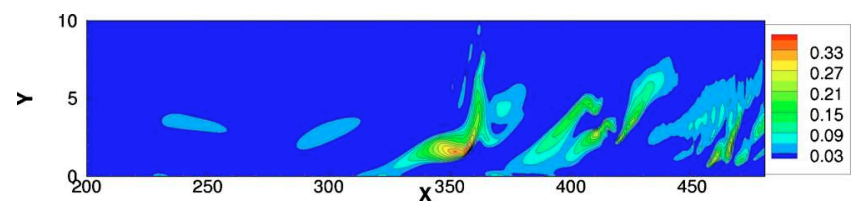

FIG. 29. (Color online) Contours of the spanwise vorticity of the perturbation in the plane $z=25$ extracted at $t=1000$ from the DNS initialized by the optimal perturbation with amplitude $A_{0}=10^{-4}$. 
wise vorticity perturbation at $t=1000$. Such results strongly recall the ones provided in Refs. 37 and 36 for the transition scenario via secondary instability of TS waves. Indeed, the TS packets convected by the separated bubble experience secondary instability and transition via the formation of $\Lambda$-vortices, which are often recovered in correspondence of the velocity spikes in the late stage of $K$-type transition. ${ }^{36}$ Such vortical structures have been identified here using the isosurfaces of the Q-criterion, i.e., the positive values of the quantity

$$
Q=\frac{1}{2}\left(\|\Omega\|^{2}-\|S\|^{2}\right),
$$

$S$ being the rate-of-strain tensor and $\Omega$ the vorticity tensor. ${ }^{38}$ In Fig. 30, showing the Q-criterion isosurfaces at $t=600$, two pairs of $\Lambda$-vortices are recovered. They are followed by a second pair of $\Lambda$-vortices (not shown) aligned with the first one, confirming that the primary transition is a typical $K$-type transition. Finally, at such low Reynolds number, transition via TS waves is not sustained, so that for $t>1000$, the flow relaminarizes.

It is noteworthy that a similar scenario has also been recovered when the flow is perturbed by a time-continuous three-dimensional random white noise at the inlet points, as it may happen in real experiments, where the inlet flow may be affected by some noise. In such a case, the $K$-type transition is sustained also for disturbances of smaller amplitude. Thus, it is possible to infer that the second type of transition arising at large times as a consequence of the onset of mode $M_{S}$ would not be recovered in an experimental framework in the presence of noise, since transition would directly occur via the $K$-type route. This could explain the difficulties arising in recovering the three-dimensional mode $M_{S}$ in experiments, its onset being observed only in low-level-of-noise environments. ${ }^{13}$

\section{Transition via Gortler modes breakdown}

In this section, the secondary transition at large times will be analyzed in detail. In Fig. 27 one can observe the onset of mode $M_{S}$ for $t>2000$ and its successive saturation (the slope of the energy gain curve decreases with time for $2000<t<8000$ ), followed by an unsteady behavior for $t>8000$. Figures 31 (a) and 31(b) show two snapshots of the spanwise vorticity isosurfaces at $t=8500$ and 9500 , respectively. At $t=8500$, one can observe roll structures, spatially extended in the streamwise direction, which strongly recall mode $M_{G}$, shown in Fig. 5(b). Later, the flow is clearly transitioning toward turbulence, since an unsteady behavior begins to be established [see Fig. 31(b)]. In particular, transition occurs in a narrow region localized in the spanwise direction, although extended in the streamwise direction from the reattachment point throughout the attached boundary layer. Figures 32(a) and 32(b) provide the streamwise velocity perturbation contours at $t=8500$ on the planes $z=36$ and 26, respectively. The shaded contours have been obtained by the DNS, whereas the solid-line contours have been obtained by the global eigenvalue analysis. Strong similarities can be observed between the two solutions; in Fig. 32(a) a great part of the energy is concentrated at the exterior

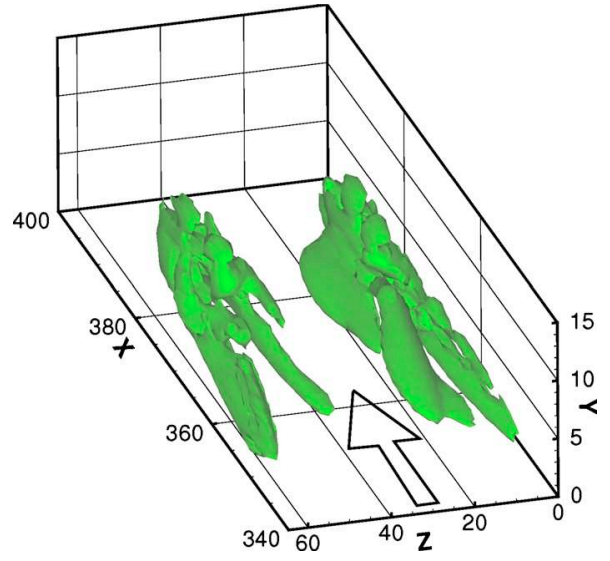

FIG. 30. (Color online) Isosurfaces of the Q-criterion identifying vortical structures extracted at $t=600$ from the DNS initialized by the optimal perturbation with amplitude $A_{0}=10^{-4}$.

of the recirculation bubble, indicating the presence of a structure very similar to mode $M_{G}$. Such a structure follows the high shear region along the separation streamline and develops in a spatially extended streamwise vortex. The presence of mode $M_{G}$ thus implies the selection of the most amplified Gortler spanwise wavenumber which is higher than the one associated with mode $M_{S}$, as previously demonstrated, explaining the concentration of the vorticity perturbation in a narrow zone in the spanwise direction. It is noteworthy that unsteadiness is recovered on a narrow region of similar spanwise extent also when a larger spanwise dimension of the
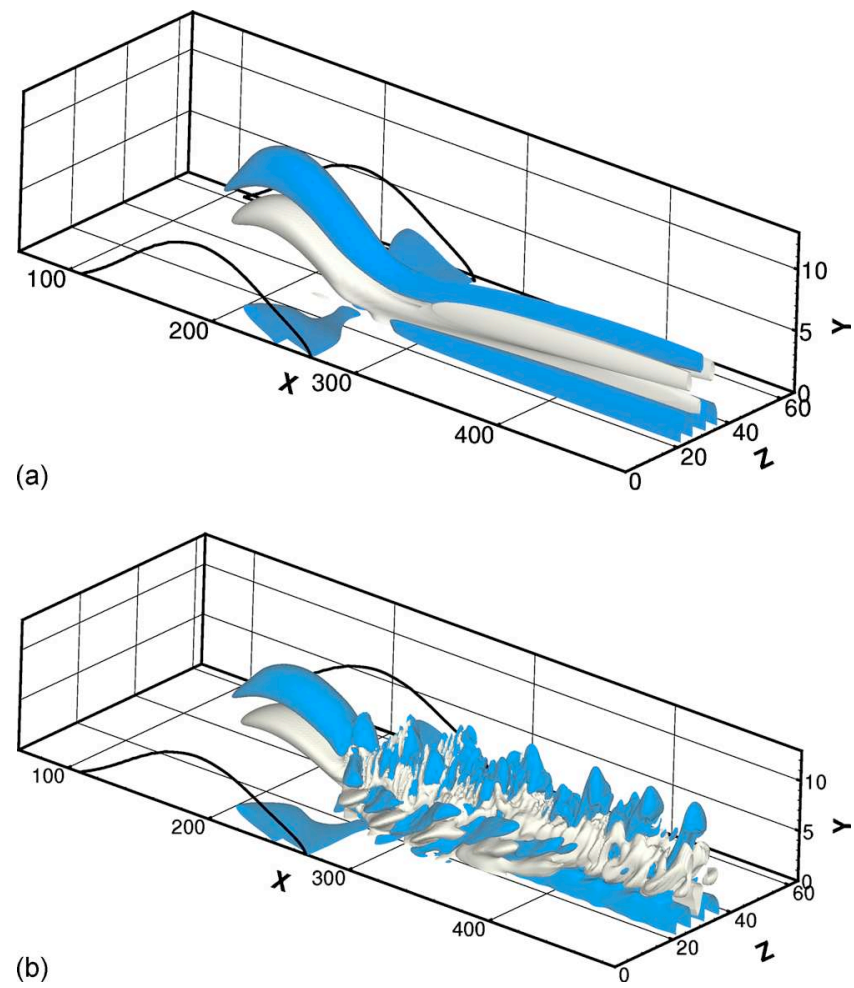

FIG. 31. (Color online) Isosurfaces of the spanwise vorticity of the perturbation extracted from the DNS at (a) $t=8500$ and (b) $t=9500$. The dark and light surfaces represent the -0.03 and 0.03 values, respectively. The solid line is the separation streamline. 

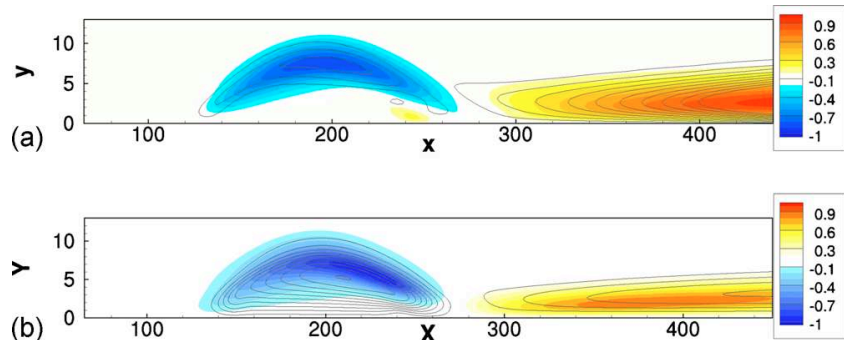

FIG. 32. (Color online) Contours of the streamwise perturbation: (a) solution extracted from the DNS at $t=8500$ and $z=36$ (shaded contours) compared with mode $M_{G}$ obtained by the global model (solid lines); (b) solution extracted from the DNS at $t=8500$ and $z=26$ (shaded contours) compared with mode $M_{S}$ obtained by the global model (solid lines). In both cases the solid lines and the shaded contours represent 14 equally spaced values from -1 to 1 ; the perturbations have been normalized with respect to their maximum value.

computational domain is chosen. Thus, it seems that the Gortler mode, although asymptotically stable, is highly spatially amplified due to its convective amplifier nature, so that it is able to reach large amplitudes leading the flow to unsteadiness.

In Fig. 32(b) one can observe some structures recalling mode $M_{S}$, the perturbation being mostly located within the bubble. Thus, as shown by the contours of Figs 32(a) and 32(b), both modes $M_{S}$ and $M_{G}$ are recovered within the flow and play a role in the transition scenario. In particular, it is possible to classify mode $M_{S}$ as a resonator ${ }^{12}$ placed within the separated zone, which is able to generate a self-sustained perturbation cycle, whereas mode $M_{G}$ can be considered the amplifier ${ }^{12}$ of the perturbation produced by the resonator, so that transition occurs via a convective mechanism as a result of the interaction of both modes. Moreover, the cycle of interaction between the two modes appears to be promoted by nonlinear interactions due to the saturation of the energy that does not allow mode $M_{S}$ to grow asymptotically.

In order to investigate in which way the Gortler mode fed by the steady resonator $M_{S}$ experiences transition, the streamwise perturbation velocity is reported in Fig. 33 in the plane $y=1$ at several instants of time. Figure 33(a) shows the presence of streamwise elongated structures at $t=4500$ due to the onset of modes $M_{S}$ and $M_{G}$. At $t=8500$ [see Fig. 33(b)] the elongated structure having positive streamwise velocity (red contours) splits into two parts in the spanwise direction, followed by an injection of negative streamwise velocity (blue contours) from the reattachment point along the region between the two high-speed elongated structures. Therefore, three pairs of streamwise vortices are created inside the attached zone. One could conjecture that this is due to nonlinear effects which are able to produce subharmonics of the dominant wavenumber. Moreover, it could also be conjectured that since modes $M_{S}$ and $M_{G}$ present a different wall-normal and spanwise distribution of the regions with positive and negative spanwise vorticity, the interaction of these modes could easily induce the generation of spanwisealternated zones of positive and negative spanwise vorticity, as shown in Fig. 31. At $t=9500$, the elongated structures seem to experience secondary instability [Fig. 33(c)] due to
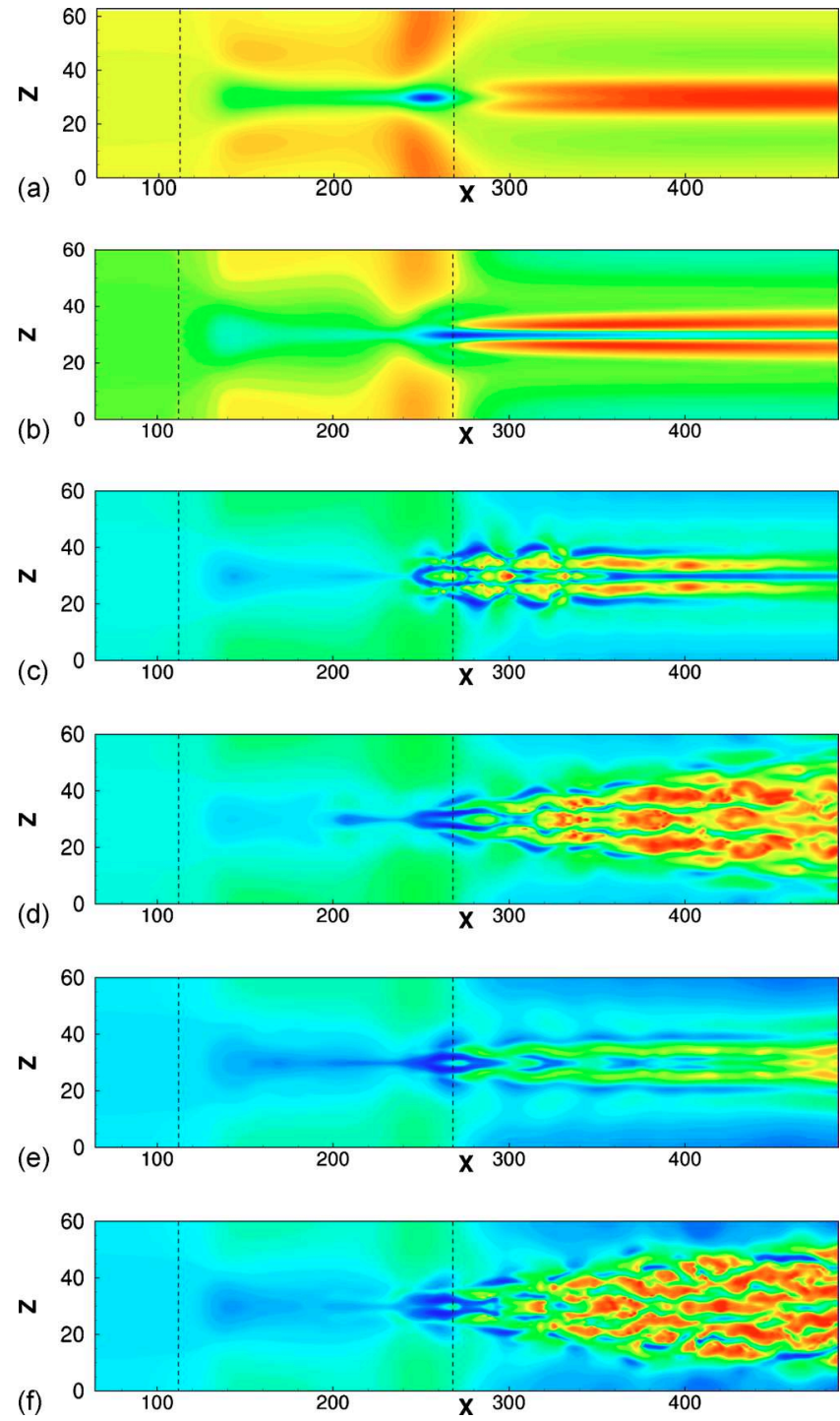

FIG. 33. (Color online) Streamwise perturbation extracted from the DNS initialized with the optimal perturbation of amplitude $10^{-4}$ at $y=1$ and (a) $t=4500$, (b) $t=8500$, (c) $t=9500$, (d) $t=10500$, (e) $t=11000$, and (f) $t=12000$. The dashed lines represent the separation and reattachment abscissas.

the close interaction of low- and high-momentum regions in the spanwise and wall-normal direction. In particular, the low-velocity structure at the middle of the spanwise domain experiences symmetric streamwise oscillations. Secondary instability suddenly evolves into a more complex pattern, the unsteadiness spreading out through the attached boundary layer, as shown in Fig. 33(d). Structures of smaller scale are recovered into the flow, which are spread out in the spanwise direction. Eventually, such a pattern does not evolve into turbulence, but the perturbation temporarily decreases and the flow seems to partially relaminarize, as shown in Fig. 33(e). At larger times, a further increase of the energy associated with a strong unsteady behavior of the flow is observed, as provided in Fig. 33(f) for $t=12000$.

In order to investigate the primary cause of the breakdown of such elongated structures, the isosurfaces of the Q-criterion (defined in section IVC 1) have been analyzed at $t=10000$ when the Gortler rolls are about to 


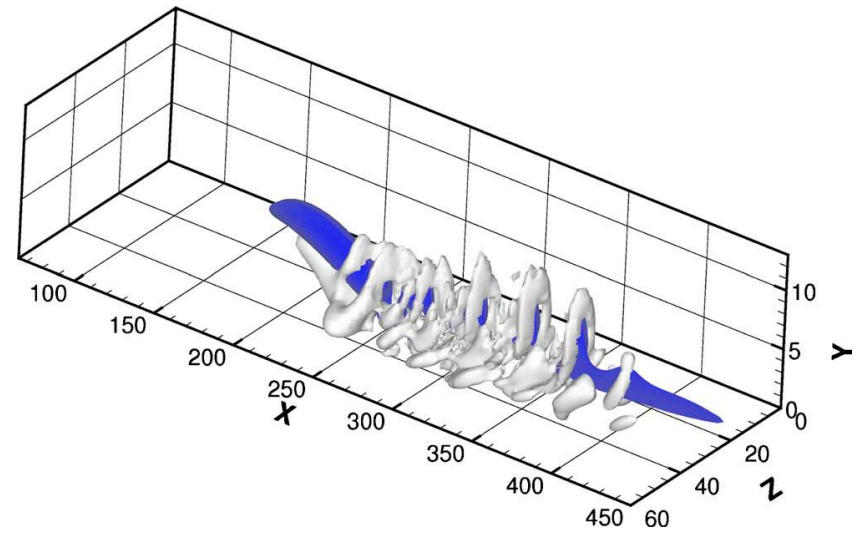

FIG. 34. (Color online) Isosurfaces of the vortical structures identified by the Q-criterion (light surfaces, $Q=6$ ) and of the negative streamwise perturbation (dark surfaces, $u=-0.2$ ) at $t=10000$.

break down. As shown in Fig. 34 by the light isosurfaces, a series of hairpin vortices are clearly identified at this stage, all of them presenting a low-momentum region between their legs (the dark surface represents a negative streamwise perturbation). Indeed, evidence of hairpin structures has been experimentally ${ }^{13,39}$ and theoretically (see Ref. 40 for instance) found for a quasivaricose secondary instability of Gortler vortices. Figure 35 shows the shaded contours of the streamwise component of the perturbation at $t=10000$ on the plane $x=260$. One can observe the mushroom-like structure typical of nonlinearly saturated Gortler vortices which is distorted on the peak of the low-speed region as well as on both sides of the stem region. Such regions correspond to the ones where the varicose secondary mode attains its maximum value, as computed in Ref. 40 for a pair of nonlinearly saturated Gortler vortices. In Ref. 40, the authors found that the varicose secondary instability of Gortler vortices is mainly due to the Reynolds stress-conversion mechanism associated with the wall-normal rate of strain of the base flow $\partial U / \partial y$, whereas sinuous modes are mostly associated with the spanwise rate of strain, $\partial U / \partial z$. It is so likely that in the considered case, the presence of alternated Gortler vortices in the wall-normal direction (a feature of both modes $M_{S}$ and $M_{G}$ ) induces a strong wall-normal shear, generating varicose oscillations and the subsequent hairpin vortices which are the primary cause of the secondary transition in the considered flow.

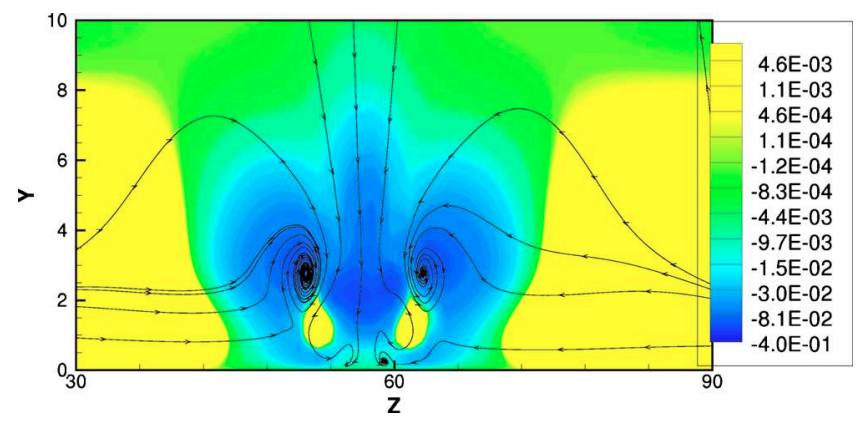

FIG. 35. (Color online) Shaded contours of the streamwise perturbation and streamlines extracted from the DNS at $t=10000$ on the $x=260$ plane.
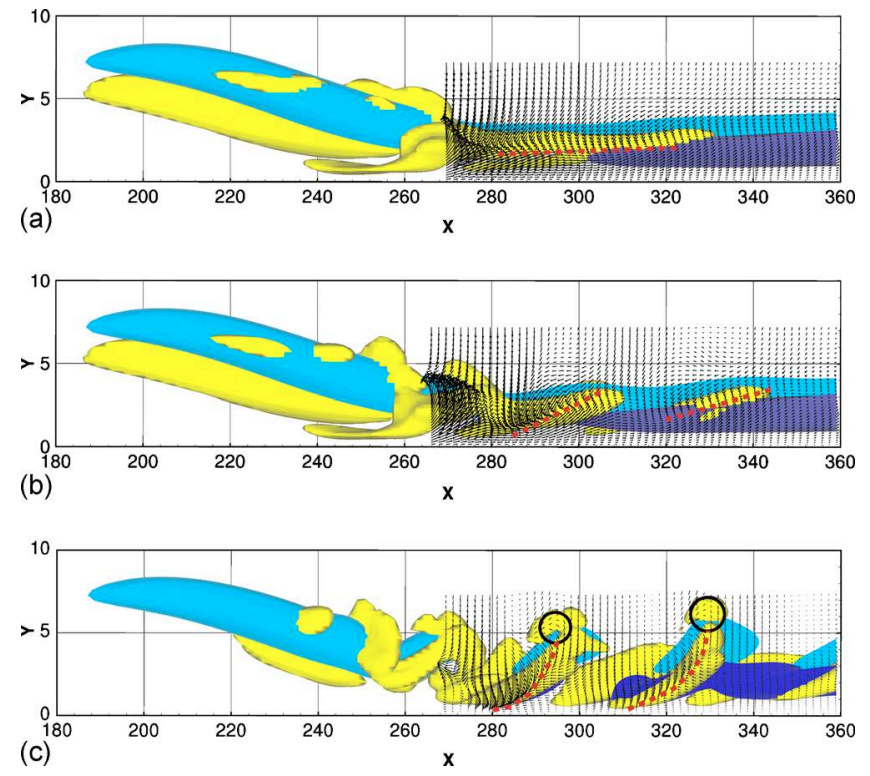

FIG. 36. (Color online) Surfaces of positive and negative streamwise perturbations (deep dark and dark surfaces correspond to $u=0.1$ and -0.1 , respectively) and of vortical structures represented by the Q-criterion (light surfaces) at (a) $t=9000$ for $Q=5$, (b) $t=9100$ for $Q=5$, and (c) $t=9300$ for $Q=20$.

Such a hypothesis has been verified by extracting snapshots of the velocity perturbation and vortical structures at different instants of time when the energy of perturbations is about to rapidly increase, namely, for $t \geq 9000$ (see Fig. 27). Figures 36(a)-36(c) provide the low- and high-speed streaks (light blue and dark blue surfaces, respectively) and the vortical structures (yellow surfaces) for $t=9000,9100$, and 9300 , respectively. The vectors are shown on the plane crossing the vortical structure between the high- and low-speed elongated structures $(z=28.5)$ for $t=9000$ and 9100 , and on the plane crossing the low-speed streak and the head of the hairpin $(z=32.5)$ for $t=9300$. The dashed line in Fig. 36(a) shows that a shear layer is created by the vertical interaction of the low- and high-speed regions, which is almost parallel to the wall. A vortical region can be observed between the two elongated structures and close to such a shear layer. A second vortical structure is also present on the opposite flank of the low-momentum zone (not shown). At $t=9100$, the elongated vortex is broken out by the wall-normal oscillations of the low-speed streak due to its secondary instability, as shown in Fig. 36(b). Thus, several inclined shear layers are created [only two of them are shown by the dashed lines in Fig. 36(b)], all of them generated by the interaction of the low- and high-momentum regions. At $t=9300$, the inclined vortices at the two flanks of the low-speed region are found to connect through vortical regions which are the "heads" of the hairpin, represented by the black circles in Fig. 36(c). Finally, a train of hairpin vortices in streamwise succession is generated, leading the flow to unsteadiness.

Thus, the present computations have shown that a secondary scenario of transition exists in a separated boundary layer flow at a low level of noise, which involves the breakdown of Gortler vortices and the generation of hairpin-like structures. It has been observed that the hairpin vortices are 
induced by the wall-normal shear layer present between the positive and negative Gortler vortices, validating the hypothesis that unsteadiness in this configuration is due to the secondary varicose instability of such elongated structures.

\section{CONCLUSIONS}

In this work the role of global centrifugal modes on the instability dynamics and the successive transition to turbulence of a separated boundary-layer flow have been investigated. The global eigenvalue analysis has been used to identify the three-dimensional centrifugal modes having the highest amplification rate. Among the others, two steady modes have been mostly analyzed: the unstable mode $M_{S}$ and the stable one $M_{G}$. A Rayleigh criterion for $M_{S}$ and a Gortler analysis for $M_{G}$ have shown that both modes have a centrifugal origin. In the former case, an intrinsic mechanism due to the curvature of the closed streamlines within the bubble originates the instability; in the latter case, the mode is characterized by a convective mechanism of Gortler type along the streamlines past the recirculation bubble.

By means of direct numerical simulations, the onset of such centrifugal modes has been investigated, considering small- or large-amplitude perturbations. Both modes $M_{S}$ and $M_{G}$ have been recovered in the flow when it is perturbed with small amplitude perturbations. In particular, using a structural sensitivity analysis, it has been shown that the stable mode $M_{G}$ could be excited by the convection of $\mathrm{KH} / \mathrm{TS}$ waves along the shear layer, which is a typical mechanism inducing a strong transient energy amplification.

In the case of large amplitude initial perturbations, two different scenarios of transition have been observed. The first scenario, which strongly recalls the $K$-type transition, is transient and is based on the strong amplification and secondary instability of $\mathrm{KH} / \mathrm{TS}$ waves. The second one, which is asymptotic, is due to a convective mechanism resulting from the interaction of modes $M_{S}$ and $M_{G}$. In particular, it has been observed that a self-generation cycle of the perturbations is established; mode $M_{S}$ has the role of a resonator, placed within the separated zone, which is able to continuously sustain perturbations in the shear layer, exciting mode $M_{G}$ which behaves as an amplifier. Thus, Gortler rolls on the attached boundary layer are created and sustained, which undergo secondary varicose instability due to the wall-normal shear induced by the interaction of low- and high-momentum regions. Such a mechanism induces the formation of a train of hairpin vortices in streamwise succession, which are often observed in the presence of Gortler vortices and which lead the flow to transition.

Thus, stable and unstable centrifugal modes have shown to play an important role in the destabilization and large-time transition of the separated flow under consideration. The scenario of transition via breakdown of Gortler rolls has been found to involve the formation of hairpin vortices in streamwise succession, which are a recurrent structure in transitional boundary layer flows (see Ref. 41). Future works will assess whether similar mechanisms of transition characterize other base flows, such as geometry-induced separation bubbles.

\section{ACKNOWLEDGMENTS}

This work was performed using HPC resources from GENCI-CCRT/IDRIS. The authors would like to thank R. Verzicco for providing the DNS code and M. Napolitano for making possible the cooperation between the two laboratories in which this work has been developed. This research has been supported by MIUR Italy and Politecnico di Bari under Contract Nos. CofinLab2001 and PRIN2007.

\section{APPENDIX A: GLOBAL EIGENVALUE ANALYSIS OPERATORS}

The global instability analysis is based on the following eigenvalue problem:

$$
\left(\mathbf{A}-i \omega_{k} \mathbf{B}\right) \hat{\mathbf{q}}_{k}=\mathbf{0}, \quad k=1, \ldots, N,
$$

where the operators $\mathbf{B}$ and $\mathbf{A}$ are defined as follows:

$$
\begin{aligned}
& \mathbf{B}=\left(\begin{array}{llll}
1 & 0 & 0 & 0 \\
0 & 1 & 0 & 0 \\
0 & 0 & 1 & 0 \\
0 & 0 & 0 & 0
\end{array}\right), \\
& \mathbf{A}=\left(\begin{array}{cccc}
\mathcal{C}_{1}-\mathcal{C}_{2}+\frac{\partial U}{\partial x} & \frac{\partial U}{\partial y} & 0 & \frac{\partial}{\partial x} \\
\frac{\partial V}{\partial x} & \mathcal{C}_{1}-\mathcal{C}_{2}+\frac{\partial V}{\partial y} & 0 & \frac{\partial}{\partial y} \\
0 & 0 & \mathcal{C}_{1}-\mathcal{C}_{2} & i \beta \\
\frac{\partial}{\partial x} & \frac{\partial}{\partial y} & i \beta & 0
\end{array}\right),
\end{aligned}
$$

with the term $\mathcal{C}_{1}=U \partial / \partial x+V \partial / \partial y$ which represents the effect of the advection of the perturbation by the base flow and $\mathcal{C}_{2}=\left(\partial^{2} / \partial x^{2}+\partial^{2} / \partial y^{2}-\beta^{2}\right) /$ Re which models the viscous diffusion effects.

\section{APPENDIX B: GORTLER EQUATIONS}

For the Gortler analysis the formalism in Ref. 30 has been followed. A local cylindrical coordinate system $(\bar{x} ; \bar{y} ; \bar{z})$ has been defined along a streamline $\phi_{G}$ around the separated zone, namely,

$$
\left\{\begin{array}{l}
\bar{x}=\Theta R \\
\bar{y}=(r-R) \sqrt{\mathrm{Re}} \\
\bar{z}=z \sqrt{\mathrm{Re}},
\end{array}\right.
$$

where $R$ is the local radius of curvature, $\Theta$ the azimuthal coordinate, and $r$ is the radial one. The variables $\bar{y}$ and $\bar{z}$ have been scaled with the square root of the Reynolds number in order to account for the different scaling of the boundary layer coordinates; for the same reason, the following nondimensional velocities have been defined:

$$
(\bar{u}, \bar{v}, \bar{w})=\left(u_{\Theta}, v_{r} \sqrt{\operatorname{Re}}, w \sqrt{\operatorname{Re}}\right),
$$

where $u_{\Theta}$ and $v_{r}$ are the velocity components along the azimuthal and radial coordinates, respectively. Since the insta- 
bility is expected to be steady and three-dimensional, the perturbation has been considered sinusoidal in the spanwise direction, namely,

$$
(\bar{u}, \bar{v}, \bar{w}, \bar{p})=[\widetilde{u} \cos (\beta z), \widetilde{v} \cos (\beta z), \widetilde{w} \sin (\beta z), \widetilde{p} \cos (\beta z)] .
$$

By assuming that the instability has an inviscid nature and that the curvature is small, one can obtain the linearized equations governing the evolution of a perturbation. Indicating with $\bar{U}$ and $\bar{V}$ the velocity components of the twodimensional base flow, one has

$$
\begin{aligned}
& \frac{\partial \tilde{u}}{\partial \bar{x}}+\frac{\partial \widetilde{v}}{\partial \bar{y}}+\beta \widetilde{w}=0 \\
& \bar{U} \frac{\partial \widetilde{u}}{\partial \bar{x}}+\frac{\partial \bar{U}}{\partial \bar{x}} \widetilde{u}+\bar{V} \frac{\partial \widetilde{u}}{\partial \bar{y}}+\frac{\partial \bar{U}}{\partial \bar{y}} \widetilde{v}=\frac{\partial^{2} \widetilde{u}}{\partial \bar{y}^{2}}-\beta^{2} \widetilde{u} \\
& \bar{U} \frac{\partial \widetilde{v}}{\partial \bar{x}}+\frac{\partial \bar{V}}{\partial \bar{x}} \widetilde{u}+\bar{V} \frac{\partial \widetilde{v}}{\partial \bar{y}}+\frac{\partial \bar{V}}{\partial \bar{y}} \widetilde{v}+\frac{\partial \widetilde{p}}{\partial \bar{y}}+G \bar{U} \widetilde{u}=\frac{\partial^{2} \widetilde{v}}{\partial \bar{y}^{2}}-\beta^{2} \widetilde{v},
\end{aligned}
$$

$$
\bar{U} \frac{\partial \widetilde{w}}{\partial \bar{x}}+\frac{\partial \bar{U}}{\partial \bar{x}} \widetilde{w}+\bar{V} \frac{\partial \widetilde{w}}{\partial \bar{y}}+\frac{\partial \bar{V}}{\partial \bar{y}} \widetilde{w}-\beta \widetilde{p}=\frac{\partial^{2} \widetilde{w}}{\partial \bar{y}^{2}}-\beta^{2} \widetilde{w},
$$

where $G=2 \delta^{*} \sqrt{\operatorname{Re}} / R$ is the Gortler number, characterizing the influence of the curvature with respect to the viscous effects. For computational purposes, the variables $\bar{p}$ and $\bar{w}$ have been eliminated. Following Ref. 30, an equation for $\bar{u}$ and $\bar{v}$ has been recovered from Eqs. (B4a), (B4c), and (B4d), whereas Eq. (B4b) has been left untouched. The system has been thus reduced to a pair of equations for the variables $\bar{u}$ and $\bar{v}$, which have been integrated marching in the $\bar{x}$ direction by a fourth-order-accurate Runge-Kutta scheme. The space marching has been initialized at $x_{0_{G}}=198$, where the inlet wall-normal and streamwise components of the perturbations have been chosen to be equal to the velocity profiles extracted from mode $M_{G}$ computed by the global eigenvalue analysis.

${ }^{1}$ P. J. Schmid, "Nonmodal stability theory," Annu. Rev. Fluid Mech. 39, 129 (2007)

${ }^{2}$ J. M. Chomaz, "Global instabilities in spatially developing flows: Nonnormality and non linearity," Annu. Rev. Fluid Mech. 37, 357 (2005).

${ }^{3}$ K. M. Butler and B. F. Farrell, "Three-dimensional optimal perturbations in viscous shear flow," Phys. Fluids A 4, 1637 (1992).

${ }^{4}$ L. Trefethen, A. Trefethen, S. Reddy, and T. Driscoll, "Hydrodynamic stability without eigenvalues," Science 261, 578 (1993).

${ }^{5} \mathrm{M}$. T. Landahl, "A note on an algebraic instability of inviscid parallel shear flows," J. Fluid Mech. 98, 243 (1980).

${ }^{6} \mathrm{C}$. P. Jackson, "A finite-element study of the onset of vortex shedding in flow past variously shaped bodies," J. Fluid Mech. 182, 23 (1987).

${ }^{7}$ A. Zebib, "Stability of viscous flow past a circular cylinder," J. Eng. Math. 21, 155 (1987).
${ }^{8}$ E. Åkervik, J. Hoepffner, U. Ehrenstein, and D. S. Henningson, “Optimal growth, model reduction and control in a separated boundary-layer flow using global eigenmodes," J. Fluid Mech. 579, 305 (2007).

${ }^{9}$ U. Ehrenstein and F. Gallaire, "Global low-frequency oscillations in a separating boundary-layer flow," J. Fluid Mech. 614, 315 (2008).

${ }^{10}$ S. Cherubini, J.-C. Robinet, and P. De Palma, "The effects of nonnormality and non-linearity of the Navier-Stokes operator on the dynamics of a large laminar separation bubble," Phys. Fluids 22, 014102 (2010).

${ }^{11}$ H. Blackburn, D. Barkley, and S. Sherwin, "Convective instability and transient growth in flow over a backward-facing step," J. Fluid Mech. 603, 271 (2008).

${ }^{12}$ O. Marquet, D. Sipp, J.-M. Chomaz, and L. Jacquin, "Amplifier and resonator dynamics of a low-Reynolds-number recirculation bubble in a global framework," J. Fluid Mech. 605, 429 (2008).

${ }^{13}$ J.-F. Beaudoin, O. Cadot, J.-L. Aider, and J. E. Wesfreid, "Threedimensional stationary flow over a backward-facing step," Eur. J. Mech. B/Fluids 23, 147 (2004).

${ }^{14}$ O. Marxen, M. Lang, U. Rist, O. Levin, and D. S. Henningson, "Mechanisms for spatial steady three-dimensional disturbance growth in a nonparallel and separating boundary layer," J. Fluid Mech. 634, 165 (2009).

${ }^{15}$ F. Gallaire, M. Marquillie, and U. Ehrenstein, "Three-dimensional transverse instabilities in detached boundary layers," J. Fluid Mech. 571, 221 (2007).

${ }^{16}$ D. Barkley, G. M. Gomes, and R. D. Henderson, "Three-dimensional instability in flow over a backward-facing step," J. Fluid Mech. 473, 167 (2002).

${ }^{17}$ O. Marquet, M. Lombardi, J.-M. Chomaz, D. Sipp, and L. Jacquin, "Direct and adjoint global modes of a recirculation bubble: Lift-up and convective non-normalities," J. Fluid Mech. 622, 1 (2009).

${ }^{18}$ F. Giannetti and P. Luchini, "Structural sensitivity of the first instability of the cylinder wake," J. Fluid Mech. 581, 167 (2007).

${ }^{19}$ Y. Na and P. Moin, "The structure of wall-pressure fluctuations in turbulent boundary layers with adverse pressure gradient and separation," J. Fluid Mech. 377, 347 (1998).

${ }^{20}$ R. Verzicco and P. Orlandi, "A finite-difference scheme for the threedimensional incompressible flows in cylindrical coordinates," J. Comput. Phys. 123, 402 (1996).

${ }^{21}$ P. Schmid and D. Henningson, Stability and Transition in Shear Flows (Springer, New York, 2001).

${ }^{22}$ R. Lehoucq, D. Sorensen, and C. Yang, ARPACK Users' Guide: Solution of Large Scale Eigenvalue Problems with Implicitly Restarted Arnoldi Methods (SIAM, Philadelphia, 1997).

${ }^{23} \mathrm{~V}$. Theofilis, S. Hein, and U. Dallmann, "On the origins of unsteadiness and three dimensionality in a laminar separation bubble," Philos. Trans. R. Soc. London 358, 3229 (2000).

${ }^{24}$ L. Marino and P. Luchini, "Adjoint analysis of the flow over a forwardfacing step," Theor. Comput. Fluid Dyn. 23, 37 (2009).

${ }^{25}$ F. Alizard, S. Cherubini, and J. C. Robinet, "Sensitivity and optimal forcing response in separated boundary layer flows," Phys. Fluids 21, 064108 (2009).

${ }^{26} \mathrm{P}$. Wilson and L. L. Pauley, "Two and three dimensional large-eddy simulations of a transitional separation bubble," Phys. Fluids 10, 2932 (1998).

${ }^{27} \mathrm{D}$. Sipp and L. Jacquin, "Three-dimensional centrifugal-type instabilities of two-dimensional flows in rotating systems," Phys. Fluids 12, 1740 (2000).

${ }^{28}$ B. J. Bayly, "Three-dimensional centrifugal-type instabilities in inviscid two-dimensional flows," Phys. Fluids 31, 56 (1988).

${ }^{29}$ P. G. Drazin and W. H. Reid, Hydrodynamic Stability (Cambridge University Press, Cambridge, England, 1981).

${ }^{30} \mathrm{P}$. Hall, "The linear development of Gortler vortices in growing boundary layers," J. Fluid Mech. 130, 41 (1983).

${ }^{31}$ G. R. Inger, "Spanwise-periodic three-dimensional disturbances in the wake of a slightly stalled wing," AIAA Paper No. 87-0456, 1987.

${ }^{32}$ F. Alizard and J.-C. Robinet, "Spatially convective global modes in a boundary layer," Phys. Fluids 19, 114105 (2007).

${ }^{33}$ S. Cherubini, J.-C. Robinet, A. Bottaro, and P. De Palma, "Optimal wave packets in a boundary layer and initial phases of a turbulent spot," J. Fluid Mech. 656, 231 (2010).

${ }^{34} \mathrm{P}$. Corbett and A. Bottaro, "Optimal perturbations for boundary layers subject to stream-wise pressure gradient," Phys. Fluids 12, 120 (2000).

${ }^{35}$ A. Monokrousos, E. Akervik, L. Brandt, and D. S. Henninson, "Global three-dimensional optimal disturbances in the Blasius boundary-layer flow 
using time-steppers," J. Fluid Mech. 650, 181 (2010).

${ }^{36} \mathrm{U}$. Rist and H. Fasel, "Direct numerical simulation of controlled transition in a flat-plate boundary layer," J. Fluid Mech. 298, 211 (1995).

${ }^{37} \mathrm{U}$. Rist and U. Maucher, "Direct numerical simulation of 2-D and 3-D instability waves in a laminar separation bubble," Report No. AGARDCP-551, 1994.

${ }^{38}$ J. C. R. Hunt, A. Wray, and P. Moin, "Eddies, stream, and convergence zones in turbulent flows," Center for Turbulence Research Report No. CTR-S88, 1988.
${ }^{39}$ J. D. Swearingen and R. F. Blackwelder, "The growth and breakdown of streamwise vortices in the presence of a wall," J. Fluid Mech. 182, 255 (1987).

${ }^{40} \mathrm{X}$. Yu and J. T. C. Liu, "On the mechanism of sinuous and varicose modes in three-dimensional viscous secondary instability of nonlinear Gortler rolls," Phys. Fluids 6, 736 (1994).

${ }^{41} \mathrm{X}$. Wu and P. Moin, "Direct numerical simulation of turbulence in a nominally-zero-pressure gradient flat-plate boundary layer," J. Fluid Mech. 630, 5 (2009). 